\title{
Contractual Structure and Wealth Accumulation $^{1}$
}

\author{
Dilip Mookherjee ${ }^{2}$ and Debraj Ray ${ }^{3}$
}

Preliminary Draft; January 2000

\begin{abstract}
This paper examines incentives of poor agents to escape poverty by saving. Owing to limited liability, low wealth creates borrowing constraints, preventing the poor from being able to finance productive projects. Future wealth increases resulting from current saving would relax these borrowing constraints, raising future productivity and incomes, thus providing a possible channel of upward mobility. However, the extent to which these benefits accrue to the agents themselves depends on the allocation of bargaining power with their lenders (or landlords). If agents have no bargaining power, the returns to saving of poor agents are appropriated entirely by lenders, resulting in poverty traps. In this case the long run wealth distribution becomes polarized into two classes, with no middle class and no interclass mobility. If on the other hand the agents have all the bargaining power then the returns to saving accrue to them entirely, and agents accumulate wealth indefinitely irrespective of initial conditions.
\end{abstract}

\footnotetext{
${ }^{1}$ This paper is based on an earlier paper titled 'Tenancy, Saving Incentives and Wealth Dynamics'. We thank seminar participants in Cornell, Florida International, Illinios, London School of Economics, Yale, Wisconsin and members of the MacArthur Network Inequality and Economic Performance for useful comments. This research has been funded by the National Science Foundation (Grant no. SES-97909254) and the MacArthur Foundation.

${ }^{2}$ Boston University; dilipm@bu.edu

${ }^{3}$ New York University and Instituto de Análisis Económico; debraj.ray@nyu.edu
} 


\section{Introduction}

It is well known that one of the unfortunate consequences of poverty is that it inhibits the poor from engaging in productive work, owing to their inability to finance such projects themselves or borrow the necessary funds. It is also well understood how the allocation of bargaining power in financial contracts between poor agents and lenders (or landlords) can significantly affect their effort incentives and productivity. In the development economics literature, this has formed the theoretical basis for the productivity-enhancing role of institutional reforms such as land reforms, contract regulations, subsidized credit and public employment programs (Eswaran and Kotwal (1986), Shetty (1988), Dutta, Ray and Sengupta (1989), Mookherjee (1997a, 1997b), Banerjee, Gertler and Ghatak (1997), and Hoff and Lyon (1995)). In the literature on macroeconomics and finance, such models have been used to explain the presence of borrowing constraints, why external finance is frequently more expensive than internal finance and why distribution may have a role in explaining output fluctuations (Bernanke and Gertler (1989), Galor and Zeira (1993), Hoff (1994), Holmstrom and Tirole (1994), Aghion and Bolton (1997) and Piketty (1997)). Sim-

ilar models have been used to explain why forms of industrial organization may depend on wealth inequality, and why worker cooperatives may occasionally perform superior to capitalist firms (Banerjee and Newman (1993), Bowles and Gintis (1994, 1995) and Legros and Newman (1996)). In these settings, reducing the inequality of wealth or power can give rise to increased productivity and per capita output, reversing traditional notions of equality-efficiency tradeoffs. They provide instances where the Coase Theorem concerning the independence of efficiency from inequality does not apply, owing to the presence of 'transaction costs' such as moral hazard and wealth constraints.

Given the central importance of wealth constraints in these models, it is important to understand their implications for saving incentives which affect the evolution of wealth over time. If agents are restricted in their access to credit, tenancy or employment owing to limited current wealth, one would expect such agents to be motivated to save aggressively in order to ease such constraints in the future. To the extent that this is true, the economy would be characterized by substantial wealth mobility, and wealth constraints would matter 
only temporarily in any given agent's lifetime. The convergence predictions of the neoclassical growth model would then continue to be valid. Yet most economies are characterized by the presence of some agents that remain persistently poor over their lifetime. Why does poverty tend to persist for some and not others? Why do some societies tend to be characterized by more upward mobility than others? When is it the case that nonconvexities inherent in credit constraints create divergence between agents with different levels of initial wealth? These questions motivate the study of dynamic models in which wealth levels of agents evolve endogenously, depending on outcomes of current projects, current financial contracts and subsequent consumption/saving decisions made by agents. Particularly interesting are the implications of institutional characteristics such as allocation of bargaining power or historical wealth inequality on the dynamics of wealth accumulation.

The existing literature on dynamic interactions between contracting and wealth typically focuses on overlapping generations models where savings take the form of 'warm glow' bequests constituting a constant fraction of lifetime income (Banerjee and Newman (1991, 1993), Galor and Zeira (1993), Aghion and Bolton (1997), Piketty (1997)). While this greatly simplifies the dynamic analysis, it is tantamount to assuming that all agents save an exogenously fixed fraction of their income, as in Solow's neoclassical growth model. Moreover, the indifference of agents to the future fortunes of their descendants inherent in this formulation is somewhat troubling when the model generates poverty traps that might be avoided by small doses of (nonpaternalistic) altruism. ${ }^{4}$ It does not throw light on the nature of saving incentives generated for any generation within its own lifetime where savings decisions are likely to affect one's own future well-being. And analyzing the determinants of savings propensities of the poor is obviously fundamental to understanding to the patterns of wealth mobility that will emerge in any given society.

This motivates our interest in a model in which savings incentives are endogenously determined, as the outcome of dynamic optimization of intertemporal welfare by households, given their expectations concerning how their future access to credit or employment will thereby be affected. To keep the model tractable we abstract from interactions across the

\footnotetext{
${ }^{4}$ For instance, the model of Loury (1981) shows how such an approach would generate patterns of long run convergence despite the complete absence of any capital markets.
} 
decisions of different agents at any point in time (that have been studied in many of the above cited papers). Our principal focus is on the effect of the allocation of contractual bargaining power between a representative agent and financiers or asset owners (hereafter referred to as principals) that she contracts with. In any given period there is an exogenous matching process pairing agents with principals who subsequently bargain over a short period financial contract. The wealth of the agent is verifiable by the principal; consequently the contract they negotiate is conditioned on this wealth. Agents subsequently make effort decisions that affect the (random) returns from the project financed, and consumption saving decisions that affect their future wealth (with a constant lending interest rate).

We study two polar settings, one in which the agent has all the bargaining power (akin to a 'competitive' market with free entry of principals), and another in which the principal has all the bargaining power (akin to a 'monopoly' market). We do not model how the allocation of bargaining power may be endogeneously determined by deeper institutional parameters (such as asset ownership patterns, frictions in search and bargaining processes, legal rules and contractual regulations, relative degrees of impatience, or number of transacting parties on either side of the market).

Our principal result is that qualitative patterns of wealth mobility depend importantly on the allocation of bargaining power. When the principals have all the bargaining power, poverty traps emerge, as poor agents have no incentives to save at all. ${ }^{5}$ Sufficiently wealthy agents maintain wealth over time. The long run wealth distribution is completely polarized, and exhibits zero mobility. In contrast, when agents have all the bargaining power, strong incentives to save are generated. No poverty trap can exist; agents accumulate wealth indefinitely irrespective of initial conditions. While the extreme situations where one side has all the bargaining power are admittedly unrealistic, our results suggest that institutional characteristics of real economies that affect relative bargaining power will be an important determinant of savings and upward mobility.

Section 2 discusses related literature. Section 3 introduces the model. Section 4 considers

\footnotetext{
${ }^{5}$ This result can be viewed as a formalization of the idea of Bhaduri (1973) wherein rent extraction motives of landlords precipitate poverty traps for their tenants.
} 
the case where principals have all the bargaining power, while Section 5 considers the reverse situation. The Appendix includes the more technical proofs.

\section{Related Literature}

Loury (1981) - the pioneering paper on wealth dynamics with imperfect capital markets - constructed a model with a neoclassical investment technology and endogenous savings based on dynamic optimization. He demonstrated that the wealth process is ergodic, resulting in a unique long run wealth distribution, independent of initial conditions. In his model, therefore, there are no poverty traps: the neoclassical convergence results extend. A similar conclusion was obtained by Banerjee and Newman (1991) in a model where wealth constrained agents were subject to moral hazard, owing to the absence of any limited liability problems.

More recent literature has presented a variety of models with poverty traps, where long run inequality and per capita output depend on initial inequality, via effects on capacitybuilding such as nutrition (Ray and Streufert (1993)), or exogenous investment thresholds (Banerjee and Newman (1993), Galor and Zeira (1993), Mani (1997)). The investment thresholds arise from nonconvexities in the technology. The poor cannot escape from poverty traps by additional borrowing (owing to capital market imperfections), saving (precluded by the assumption of constant savings rate), or gradual accumulation of capital stock (owing to the technological nonconvexities). Piketty (1997) considers a model with a convex technology and a constant savings rate. This model has no poverty traps, but there are multiple steady states and long run output can depend on initial inequality. The model of Aghion and Bolton (1997) has a nonconvex technology and a constant savings rate high enough to ensure that wealth is ergodic; they focus on inequality dynamics in the intermediate term. Ghatak, Morelli and Sjöstrom (1997) analyse a model with an exogenous investment threshold and endogenous savings in a two period setting. They focus on the effects of credit market imperfections on effort and saving incentives of young agents in a competitive setting where agents have all the bargaining power. The model studied in this paper is contrasted to this literature in that it has a convex technology, endogenous savings decisions in 
an infinite horizon framework, and examines the implications of unequal bargaining power. Whether or not poverty traps arise depends on the endogenous emergence of investment thresholds, determined exclusively by institutional characteristics of the economy.

Other literature on the dynamics of inequality in a asymmetric information contracting framework includes Green (1987), Thomas and Worrall (1990), Atkeson and Lucas (1992), Wang (1995), and Phelan (1998). All of these papers study efficient insurance where agent endowments are private information and follow an i.i.d. process. They differ from our model in a variety of respects:

(a) the incentive problem arises from private information (unobservable endowments) rather than moral hazard (unobservable effort);

(b) agents are assumed unable to save;

(c) they use a mechanism-design approach, i.e., where a social planner devises an efficient long-term mechanism, whereas we study a sequence of equilibrium short-period contracts;

(d) they consider the case in which the planner seeks to maximize the payoff of the agents, which corresponds to the case in our model where the agents have all the bargaining power.

The results also differ markedly: the insurance model tends to generate (almost) all agents drifting down into poverty. In the corresponding 'competitive' case, our model produces a diametrically opposite conclusion: agents' wealth drift upwards indefinitely, irrespective of initial conditions. The upward drift arises owing to the strong saving incentives in our model. Another reason for the difference in results is the limited liability aspect of our model, which ensures that the incentive problem remains nonnegligible for poor agents. ${ }^{6}$

\footnotetext{
${ }^{6}$ The arguments employed in our model in the competitive case are, however, closely connected to those used in the dynamic insurance literature in some respects. This is discussed further in Section 5.
} 


\section{The Model}

\subsection{Projects and Payoffs}

There are two types of agents: $\mathrm{A}$ and $\mathrm{P}$. Time runs $0,1,2, \ldots$ At any date, a $\mathrm{P}$ is matched with an $\mathrm{A}$ with wealth $w$; the latter is an entrepreneur operating a productive project for which $\mathrm{P}$ provides finance, or leases relevant assets. A can engage in a project at a scale $\alpha$ lying between 0 and 1. At scale $\alpha$ the project involves an upfront cost of $\alpha f$, and yields a return $\alpha R$, where $R>f$. With probability $1-e_{t}$, the project fails to generate any return, where $e_{t} \in[0,1]$ is noncontractible effort of the agent at $t$. Hence there are constant returns to scale, and a capacity constraint on the scale of the project, possibly arising from limits on the time or attention of the entrepreneur. But the production technology is convex.

A's date $t$ payoff is $u\left(c_{t}\right)-D\left(e_{t}\right)$, where $c_{t} \geq 0$ is A's consumption at $t . u$ is strictly increasing and concave, with $u(0)=0$; so linear utility is possible. Note also that no assumption has been made concerning marginal utility at zero consumption. Effort disutility $D$ is strictly increasing, strictly convex, satisfying $D(0)=D^{\prime}(0)=0, \lim _{e \rightarrow 1} D(e)=\infty$, and $D^{\prime \prime \prime}(e) \geq 0$ for all $e \geq 0$.

Once matched at a given date, $\mathrm{P}$ and $\mathrm{A}$ enter into a short-term contract, where $\mathrm{P}$ pays for setup cost $\alpha f$ and receives payments $q_{t}, r_{t}$ from $\mathrm{A}$ in the event of failure and success respectively. The resulting wealth of $\mathrm{A}$ at the end of date $t$ is then $x_{t}=w_{t}-q_{t}$ in failure state, and $y_{t}=w_{t}+\alpha R-r_{t}$ in the success state. Equivalently, the contract can be represented by $x_{t}, y_{t}$, with resulting transfers to $\mathrm{P}$ being $w_{t}-\alpha f-x_{t}$ in failure state, and $w_{t}-\alpha f+\alpha R-y_{t}$ in the success state. The contract has to respect the limited liability constraint $x_{t}, y_{t} \geq 0$. Combined with the assumption that utility of zero consumption is

finite, this limited liability constraint has bite for agents with negligible wealth, necessitating payment of informational rents to induce effort incentives. 


\subsection{Consumption-Saving Decisions}

Let $z_{t} \in\left\{x_{t}, y_{t}\right\}$ denote A's wealth at end of period $t$. A then decides consumption $c_{t} \in$ $\left[0, z_{t}\right]$. The resulting saving $z_{t}-c_{t}$ is invested at an exogenous rate of return $r>0$, resulting in a level of wealth $w_{t+1}=(1+r)\left(z_{t}-c_{t}\right)$ at the beginning of date $t+1$, whence $\mathrm{A}$ is matched again with a $\mathrm{P}$.

A's objective is to maximize $\sum_{t=1}^{\infty} \delta^{t-1} u\left(c_{t}\right)-D\left(e_{t}\right)$, with discount rate $\delta=\frac{1}{1+r}$. The assumption on the discount rate ensures that in autarky A would maintain his wealth, so that the wealth dynamics in our model result entirely from the effects of contractual structure. On the other hand, P's objective at $t$ is to maximize expected profit at $t$, given by $w_{t}-\alpha f+e_{t}\left(\alpha R-y_{t}\right)-\left(1-e_{t}\right) x_{t}$. This corresponds to the assumption that $\mathrm{A}$ and $\mathrm{P}$

enter into a short period contract, and the nature of the market is such that $\mathrm{A}$ is very likely to be matched with other P's at succeeding dates that it does not pay $\mathrm{P}$ to incorporate effects of the current contract on the same A's wealth at future dates. A of course knows that his future wealth will affect the nature of contracts in the future, so will incorporate these effects when making current decisions.

\subsection{The Static Benchmark}

It is useful to quickly review the case where there is a single date (so we drop the $t$ subscript in this section), studied in more detail in Mookherjee (1997a,1997b). Then A must consume the entire end-of-period wealth, resulting in the following expression for expected utility $e u(y)+(1-e) u(x)-D(e)$.

The set of feasible contracts for A with given wealth $w$ is $\alpha, x, y, e$ satisfying the following four constraints:

(i) Incentive Constraint (IC): $D^{\prime}(e)=\max \{0, u(y)-u(x)\}$

(ii) A's Participation Constraint (APC): $u(x)+e D^{\prime}(e)-D(e) \geq u(w)$

(iii) P's Participation Constraint (PPC): $w-\alpha f+e(\alpha R-y)-(1-e) x \geq 0$

(iv) Limited Liability (LL): $x, y \geq 0$ 
Note that there is always a feasible contract $\alpha=0, x=y=w, e=0$, which we hereafter call the null contract. The set of feasible contracts maps into the set of feasible payoffs $U_{P}=w-\alpha f+e(\alpha R-y)-(1-e) x, U_{A}=u(x)+e D^{\prime}(e)-D(e)$, allowing us to generate a utility possibility frontier. Constrained efficient contracts generate maximal (Pareto undominated) payoffs within the feasible set.

Lemma 1 Every nonnull feasible contract with $\alpha \in(0,1)$ is weakly Pareto dominated by one with either $\alpha=1$ or $\alpha=0$.

Proof: If $e R-f>0$ then putting $\alpha=1$ and keeping $x, y, e$ unchanged preserves feasibility, and increases the lenders payoff, while leaving the borrower's payoff unchanged. On the other hand if $e R-f<0$ then the same is true if $\alpha$ is set at 0 and $x, y, e$ are left unchanged.

Hence without loss of generality we can ignore interior values of project scale $\alpha$. Note also that since $u$ is concave, any contract with $\alpha=0$ is weakly dominated by one involving $e=0$ and $y=x$. The two participation constraints imply that the only contract satisfying $e=\alpha=0$ is the null contract. Hence the concavity of $u$ implies that if there exists a feasible non-null contract, we can focus entirely on contracts involving $\alpha=1$ when describing constrained efficient contracts.

$\mathrm{P}$ and $\mathrm{A}$ bargain over the set of constrained efficient contracts. If $\mathrm{P}$ has all the bargaining power, the contract $\alpha(w), x(w), y(w), e(w)$ maximizes $U_{P}$ subject to IC, APC, PPC and LL. In contrast, if A has all the bargaining power, $\alpha(w), x(w), y(w), e(w)$ maximizes $U_{A}$ subject to IC, APC, PPC and LL

Let us first consider the properties of the contract resulting when $\mathrm{P}$ has all the bargaining power, and $w=0$. Then APC is implied by LL and IC, so it is optimal for $P$ to set $x=0$, and the problem reduces to selecting $y(w), e(w)$ to maximize $e[R-y]$, with $D^{\prime}(e)=u(y)$. Note that it is optimal for $\mathrm{P}$ to set $e>0, y>0$, since a small increase in $y$ from 0 would increase P's profit. Let the optimal $e$ be denoted $e^{*}$. 
Assumption $[\mathbf{A}] e^{*}\left[R-u^{-1}\left(D^{\prime}\left(e^{*}\right)\right)\right]>f$.

[A] implies that with $w=0$ there exists a non-null feasible contract; hence the optimal contract from P's point of view generates positive surplus to both. A earns a positive informational rent because only the carrot (positive $y$ ) can be used to provide effort incentives, and assumption $[\mathrm{A}]$ ensures that this is in the interest of $\mathrm{P}$. This is where the limited liability assumption plays a crucial role.

If $[\mathrm{A}]$ is not satisfied, there is no non-null feasible contract for an agent with zero wealth. In that case defining $w^{*}=e^{*}\left[R-u^{-1}\left(D^{\prime}\left(e^{*}\right)\right)\right]-f>0$, there is no non-null feasible contract for all agents with wealth between 0 and $w^{*}$. Such agents will therefore be excluded from the market altogether. If $e^{*} D^{\prime}\left(e^{*}\right)-D\left(e^{*}\right) \geq u\left(w^{*}\right)$ there is a non-null feasible contract for a $w^{*}$ agent. If the inequality is strict, then the optimal contract for $\mathrm{P}$ for such an agent will award a strictly positive surplus to both parties: this corresponds to a phenomenon analogous to a tenancy ladder, where agents must accumulate a certain minimal level of wealth before they can gain access to the market. In what follows we shall exclude this interesting case.

Previous literature shows how the allocation of bargaining power or A's wealth affects the contract and the induced effort. For instance when $U$ is linear then $e_{P}(w) \leq e_{A}(w) \leq$ $e^{F} \equiv D^{\prime-1}(R)$, and both $e_{P}(w), e_{A}(w)$ are nondecreasing (Mookherjee (1997a)). Increased wealth permits agents to post larger collateral, and thus credibly commit to accepting larger punishments in case the project fails. This induces higher effort levels. Moreover, given assumption $[\mathrm{A}]$, poor agents must be offered informational rents that are increasing in the level of effort that has to be induced. So the allocation of bargaining power matters: these rents are counted as a benefit by A but a cost by P. Shifting bargaining power to $\mathrm{P}$ thus reduces the level of effort induced. How these results are modified in the case where $\mathrm{A}$ is risk-averse is analysed in Mookherjee (1997b). 


\subsection{Infinite Horizon Markov Equilibria}

We now move to the infinite horizon setting, and focus on Markov equilibrium, where contracts are conditioned on the wealth of agents: $\alpha(w), x(w), y(w), e(w)$, combined with A's consumption strategy $c(z)$. Let $V(w)$ denote the resulting present value utility for A at the beginning of any date when he has wealth $w$.

Then A's consumption strategy is as follows. Let $w^{\prime}$ denote his target initial wealth for the next date. This corresponds to saving $\delta w^{\prime}$. The target wealth solves the following problem.

$$
B(z) \equiv \max _{0 \leq \delta w^{\prime} \leq z} u\left(z-\delta w^{\prime}\right)+\delta V\left(w^{\prime}\right) .
$$

Here $B(z)$ denotes A's present value utility at any date where he attains end-of-period wealth $z$ following realization of the project return and payment of transfers to $\mathrm{P}$ in line: call this the ex post value function. This incorporates A's subsequent consumption-saving decision at this date, and what A expects to attain from the following date. This ex post value function helps define A's savings and effort incentives, to which we now turn.

The saving incentive constraint can be written as

(SIC): For all $z \geq 0: c(z)=z-\delta w(z)$, where $w(z)$ solves (1).

while $A$ 's effort incentive under any given contract $(x, y)$ : $e$ will be chosen to maximize $e B(y)+(1-e) B(x)-D(e)$, yielding

(EIC): $D^{\prime}(e)=\max \{B(y)-B(x), 0\}$

Finally, the relation between ex ante and ex post value functions is as follows:

$$
V(w) \equiv e(w) B(y(w))+(1-e(w)) B(x(w))-D(e(w))
$$

The corresponding sequence of events is depicted in Figure 1. 


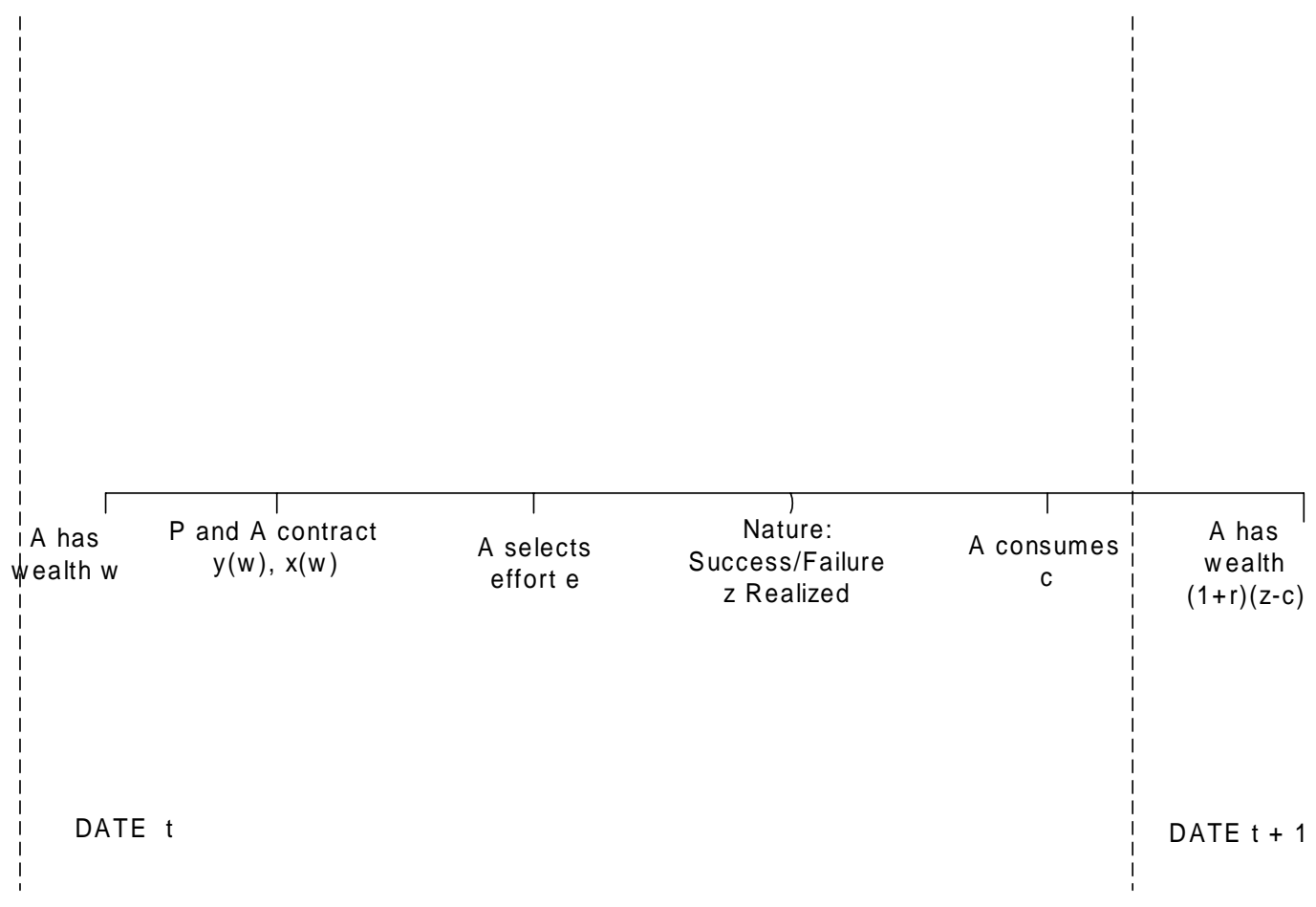

Figure 1: SEqUENCE of Events

\subsection{Feasible Contracts; Equilibrium}

Given equilibrium $\alpha(w), x(w), y(w), e(w), c(z)$ and associated value functions $B, V$, a contract $(\alpha, x, y, e)$ is feasible for $\mathrm{A}$ with wealth $w$ if it satisfies (IC), (LL), (PPC) and

(APC): $e B(y)+(1-e) B(x)-D(e) \geq B(w)$

As in the static case, this helps define the set of feasible contracts, and thereafter the set of constrained efficient contracts between this $\mathrm{A}$ and the $\mathrm{P}$ he is currently matched with. 
In the case where principals have all the bargaining power, the appropriate notion of Markov equilibrium is the following.

Definition $(\alpha(),. x(),. y(),. e(),. c()$.$) is a P-equilibrium if (a) c(z)$ satisfies (SIC) for every $z$, and (b) for every $w: \alpha(w), x(w), y(w), e(w)$ maximizes $w-\alpha f+e[\alpha R-y]-(1-e) x$ over the set of contracts feasible relative to this equilibrium.

When the allocation of bargaining power is reversed, this is modified as follows.

Definition $\{x(),. y(),. e(),. c()$.$\} is an A-equilibrium if (a) c(z)$ satisfies (SIC) for every $z$, and (b) for every $w$ : $\alpha(w), x(w), y(w), e(w)$ maximizes $e B(y)+(1-e) B(x)-D(e)$ over the set of contracts feasible relative to this equilibrium.

Note that Lemma 0 also applies to this setting. Hence we may confine our attention to contracts involving $\alpha=0$ or 1 . Note however that $B$, unlike $u$, may not be concave. So there may exist feasible contracts involving no production: $\alpha=0$, but which are non-null, with $y \neq x$. If $y<x$ then of course this is equivalent to the null contract since it will elicit $e=0$. But the case with $y>x, e>0, \alpha=0$ still remains. We however shall not consider the possibility of such contracts. There are two reasons for this. First, if $\alpha=0$ then there is no project financed, and hence no project outcomes on which transfers can be conditioned. Then $y=x$ and we are back to a null contract. Second, a non-null contract with $\alpha=0$ may arise only owing to the nonconavity of $B$ : A would optimally like to bear some risk, and so effectively purchases a lottery from P. But it an expensive way of buying risk, since it necessitates the application of costly effort by A. Such a contract would be dominated by a randomized contract with $e=0$. Given that we do not consider randomized contracts, it does not make any sense to consider these contracts either.

Hence it what follows, either no non-null feasible contract exists at $w$, in which case the equilibrium must be associated with $x(w)=y(w), e(w)=0=\alpha(w)$, or (without loss of generality) the equilibrium involves a constrained efficient contract in which $\alpha=1$. 


\section{Where $\mathrm{P}$ has all the Bargaining Power}

\subsection{The 'Floor' Contract}

The floor contract is the one offered to A with $w=0$. In what follows, remember that [A] continues to be assumed. The next proposition describes some properties of contracts offered to agents with zero wealth: specifically these are similar to optimal contracts in the static setting in that $\mathrm{A}$ will receive informational rents.

Proposition 1 In any P-equilibrium:

(a) $e(0)>0, y(0)>0, x(0)=0$.

(b) $V(0)=\frac{e(0) D^{\prime}(e(0))-D(e(0))}{1-\delta}>0$

(c) There exists $w^{*}$ such that $V(w)=V(0)>B(w)$ for all $w \in\left[0, w^{*}\right)$, and $V(w)>V(0)$ for all $w>w^{*}$.

Proof: Note that $c(0)=0=w(0)$, so

$$
B(0)=u(0)+\delta V(0)=\delta V(0)
$$

while

$$
V(0)=e(0) B(y(0))+(1-e(0)) B(x(0))-D(e(0)),
$$

where $x(0), y(0), e(0)$ maximize $e[R-y]-(1-e) x-f$ subject to (IC), (LL), (PPC) and (APC) corresponding to $w=0$.

Note that at $w=0,(\mathrm{APC})$ is implied by (LL) and (IC): $e B(y)+(1-e) B(x)-D(e)=$ $B(x)+e D^{\prime}(e)-D(e) \geq B(x) \geq B(0)$

So $y(0), x(0), e(0)$ maximizes $e[R-y]-(1-e) x$ subject to $D^{\prime}(e)=\max \{B(y)-B(x), 0\}$, $y, x \geq 0$.

We now claim that $x(0)=0$. If this is false, then $\mathrm{P}$ can lower $x(0)$ slightly and realize an improvement as long as $R-y(0)+x(0)>0$. On the other hand if $R-y(0)+x(0) \leq 0$, we have $y(0)>0$ and a slight lowering of $y(0)$ will realize an improvement. 
Hence

$$
V(0)=B(0)+e(0) D^{\prime}(e(0))-D(e(0))=\delta V(0)+e(0) D^{\prime}(e(0))-D(e(0))
$$

implying

$$
V(0)=\frac{e(0) D^{\prime}(e(0))-D(e(0))}{1-\delta}
$$

Finally we show that $y(0)>0$, which implies $V(0)>0$. If this is untrue, $y(0)=0=e(0)$ and (PPC) is violated, so there cannot exist a feasible contract at $w=0$ generating positive profit. But $\mathrm{P}$ can select $y=y^{*}(>0)$ from the static optimal contract, in response to which A will respond with $e \geq e^{*}$, since $B(y)-B(0) \geq u(y)-u(0)$ for all $y$. This will generate a profit for $\mathrm{P}$ no smaller than the static optimal contract, so assumption [A] implies that there does exist a feasible and profitable contract at $w=0$.

Now note that $B$ is a strictly increasing function, and $B(0)=\delta V(0)<V(0)$. Define $w^{*}$ $\operatorname{assup}_{w}\{w \mid V(0)>B(w)\}$. And note that for any $w>w^{*}, V(w) \geq B(w)>B\left(w^{*}\right) \geq V(0)$.

The implication of Proposition 1 is that A's value function $V$ must be nonconvex. This is evident if $B$ is continuous it must be flat (i.e., no saving incentives) over a range of low wealth levels, whereas it must be rising later on. And if $B$ is discontinuous at 0 , this necessitates a discontinuity of $V$ at 0 . Poor agents must be offered a 'floor' contract that awards them utility in excess of their outside option. This nonconvexity, which plays a fundamental role in the analysis below, arises entirely from aspects of the contractual structure, rather than the technology.

\subsection{A Simple $P$-Equilibrium}

We shall try to explicitly construct a P-equilibrium with continuous value functions (hereafter referred to as a simple P-equilibrium (SPE)), where

$$
V(w)= \begin{cases}V(0), & \text { if } w \leq w^{*} \\ B(w), & \text { if } w>w^{*}\end{cases}
$$




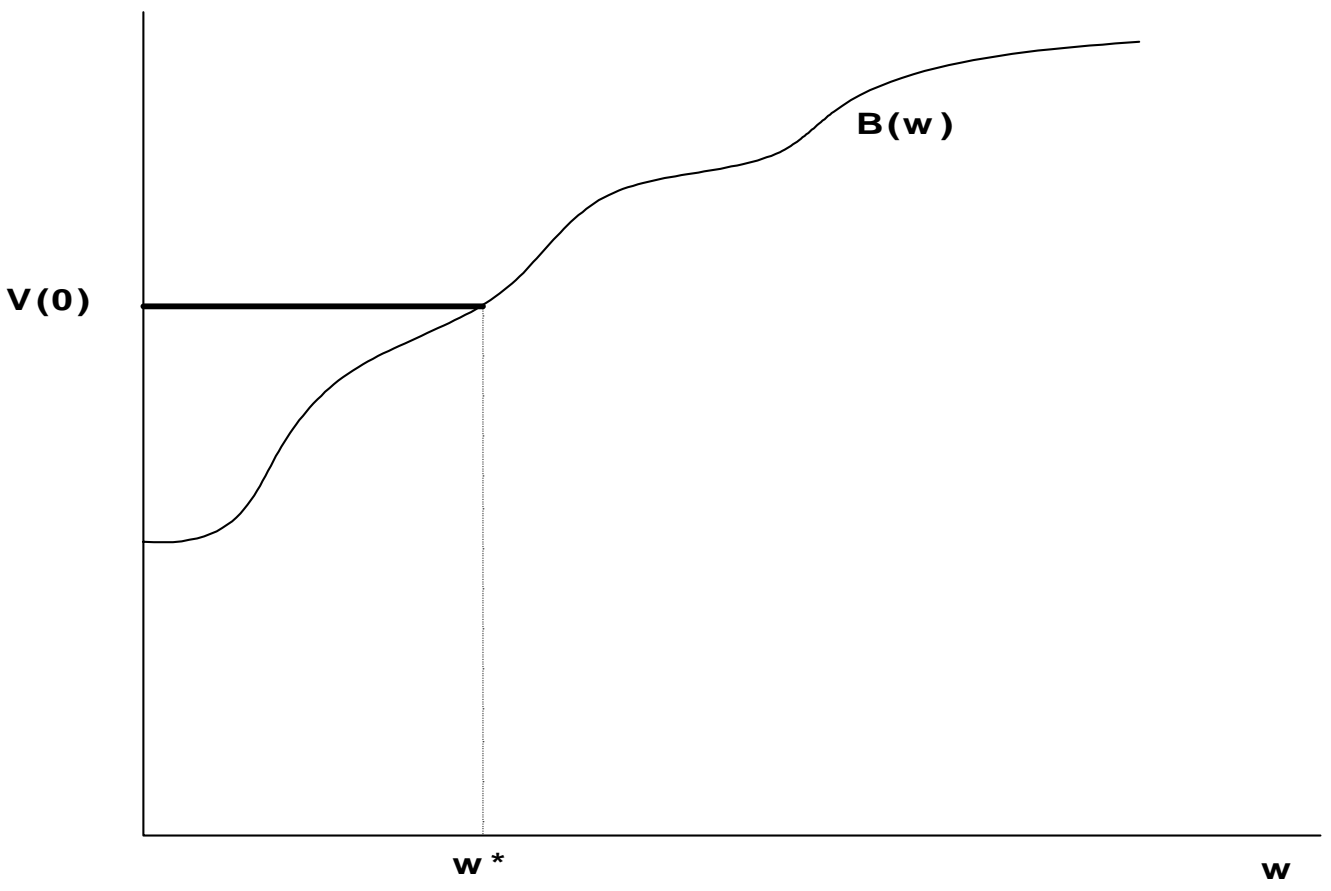

Figure 2: The Ex Ante Value Function $V$ in a Simple P-Equilibrium.

and rents are generated only for poor agents. See Figure 2. To this end we impose the following assumption ( $e^{*}$ denotes the optimal P-equilibrium effort for $w=0$ in the static setting):

Assumption [B]

$$
(1+\delta) u\left(\frac{R}{1+\delta}\right)-u(R)<\delta(1-\delta)\left[e^{*} D^{\prime}\left(e^{*}\right)-D\left(e^{*}\right)\right]
$$

[B] can be viewed as a restriction on the curvature of $u$ (given all other parameters), stating that the agent is not "too risk-averse" (it is always satisfied when $U$ is linear). Given concave $u$, it can also be interpreted as saying that $\delta$ cannot be close to 1 , given $u$ and $R$. 


\subsection{Consumption-Saving Decisions in a Simple P-equilibrium}

Note that an SPE satisfies

$$
V(w)=\max \{V(0), B(w)\}
$$

implying

$$
B(z)=\max _{0 \leq \delta w \leq z}[u(z-\delta w)+\delta \max \{V(0), B(w)\}]
$$

Contrast this with the standard Ramsey optimal saving problem, where the value function $W(w)$ satisfies:

$$
W(w)=\max _{0 \leq \delta \tilde{w} \leq w}[u(w-\delta \tilde{w})+\delta W(\tilde{w})]
$$

and $W(0)=u(0)+\delta W(0)$, so that

$$
W(0)=\frac{u(0)}{1-\delta}=0
$$

The contrasting feature of a P-equilibrium is that A has access to the floor contract at $w=0$, which offers positive surplus $V(0)>0$. Hence the consumption-saving decision of A, described by (5), differs from a standard Ramsey problem, only insofar as A always has available an exit option, i.e., of running down his wealth to 0 at any given date and exiting with the payoff $V(0)>0$.

\subsubsection{An Artificial Ramsey Problem with an Exit Option}

We can pin down the ex post value function $B$ of the agent in a SPE, using (5), which corresponds to an artificial (deterministic) Ramsey problem with the exit option $V^{*} \equiv V(0)$.

Definition $u$ satisfies the Maintenance Condition (MC) relative to $V^{*}$ if

$$
\lim _{c \rightarrow \infty}\left[u(c)-c u^{\prime}(c)\right] \geq(1-\delta) V^{*}
$$

Proposition 2 Consider the (deterministic) Ramsey problem with exit option $V^{*}$.

(i) If $u$ does not satisfy $M C$ relative to $V^{*}$, then the solution is (for all $z$ ):

$$
c(z)=z, B(z)=u(z)+\delta V^{*}
$$




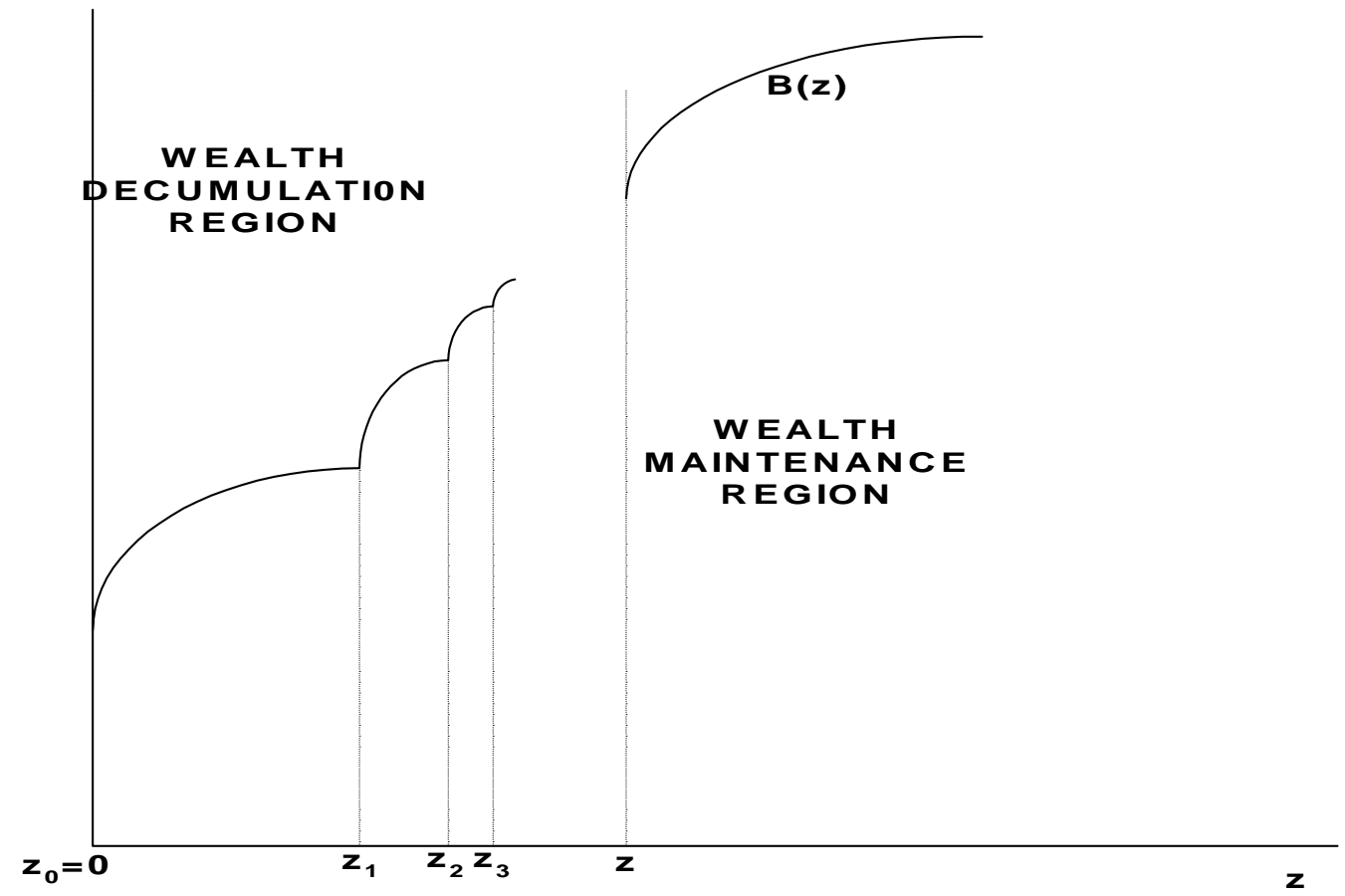

Figure 3: The Ex Post Value Function $B$.

(ii) If $u$ satisfies $M C$ relative to $V^{*}$, the solution is described as follows. Let $T(z)$ equal the optimal exit time for an agent with initial wealth $z$, with $T(z)=\infty$ if the agent never exits. There exists an infinite sequence $\left\{z_{k}\right\}_{k=0}^{\infty}$, with $0=z_{0}<z_{1}<z_{2}<\cdots$, and $z_{\infty} \equiv \lim _{k \rightarrow \infty} z_{k}<\infty$, such that $T(z)=k$ for $z \in\left[z_{k-1}, z_{k}\right]$ (with indifference holding for adjacent values of $T$ at the endpoints), and $T(z)=\infty$ for all $z \geq z_{\infty}$.

The associated value function in case (ii) is

$$
\begin{aligned}
B(z) & =\frac{1-\delta^{k}}{1-\delta} u\left(\frac{(1-\delta) z}{1-\delta^{k}}\right)+\delta^{k} V^{*} \text { for all } z \in\left[z_{k-1}, z_{k}\right] \\
& =\frac{1}{1-\delta} u((1-\delta) z) \text { for all } z \geq z_{\infty} .
\end{aligned}
$$

Proof: Consider the related problem of selecting a real number $x \in\left[1, \frac{1}{1-\delta}\right]$ to maximize $\psi(x ; z) \equiv x u\left(\frac{z}{x}\right)+[1-(1-\delta) x] V^{*}$. 
(Here $x$ corresponds to $\frac{1-\delta^{k}}{1-\delta}$, where the exit date $k$ is treated as a continuous variable in $[1, \infty)$. Since it is optimal to smooth consumption perfectly until the exit date, A will consume uptil the exit date at the steady level of $c(z)=\frac{z(1-\delta)}{\left(1-\delta^{k}\right)}$, thereby running down wealth to 0 at $k$, and exiting with $V^{*}$. This generates the value function $B(z)=\frac{1-\delta^{k}}{1-\delta} u\left(\frac{z(1-\delta)}{1-\delta^{k}}\right)+$ $\delta^{k} V^{*}$.)

Now note that

$$
\begin{aligned}
\psi_{x} & =u\left(\frac{z}{x}\right)-x u^{\prime}\left(\frac{z}{x}\right) \frac{z}{x^{2}}-(1-\delta) V^{*} \\
& =u\left(\frac{z}{x}\right)-\frac{z}{x} u^{\prime}\left(\frac{z}{x}\right)-(1-\delta) V^{*}
\end{aligned}
$$

so the concavity of $u$ implies that $\psi$ is concave in $x$, for any $z$.

If $u$ does not satisfy MC relative to $V^{*}$, then $\psi_{x}(x ; z)<0$ for all $x$, so then the optimal value of $x=1$, i.e., $k=1$. Then A consumes all current wealth and exits at the next date, implying (i).

It is easily checked the same is the case when $u$ satisfies $\mathrm{MC}$ relative to $V^{*}$ as an equality.

If $u$ strictly satisfies MC relative to $V^{*}$, there exists $m$ such that $u(m)-m u^{\prime}(m)=$ $(1-\delta) V^{*}$. Define $\bar{z}=\frac{m}{1-\delta}$. Then $z>\bar{z}$ implies $\frac{z}{x} \geq \bar{z}(1-\delta)=m$ for all $x \in\left[1, \frac{1}{1-\delta}\right]$. Hence $\psi_{x}(x ; z) \geq 0$ for all $x$, so optimal $x=\frac{1}{1-\delta}$, or $k=\infty$. Conversely, $z<\bar{z}$ implies that $\psi_{x}\left(\frac{1}{1-\delta} ; z\right)<0$, so it is optimal for the agent to exit at some finite date. In particular, $z \leq m$ implies that optimal $x=1=k$. And $z \in\left(m, \frac{m}{1-\delta}\right)$ implies that the agent must exit at some date $k>1$.

To calculate the exact exit date, the concavity of $\psi$ implies that it suffices to look at the two integer solutions for $k$ generating values of $\frac{z}{x}$ closest to $m$. Every finite $k$ will therefore be an optimal exit date for some $z$, and the exact switch points can be calculated by the condition of indifference between adjacent exit dates.

Proposition 2 identifies the value function $B$, given the floor utility $V^{*}$, in any SPE. The optimal saving strategy is characterized by an infinite sequence of thresholds $z_{1}, z_{2}, \ldots$ converging to a finite threshold $z_{\infty}$, with the property that for any wealth below $z_{1} \mathrm{~A}$ wishes to save nothing and run down his wealth to 0 , while above $z_{\infty}$ he seeks to maintain 
his wealth. For intermediate wealth levels he plans to consume at a rate which would run down his wealth in a finite number of periods. The resulting ex post value function $B$ is continuous and strictly increasing. It is concave beyond $z_{\infty}$ in the wealth maintenance region. Before this, however, every threshold constitutes a point where $B$ is kinked and locally nonconvex. Crossing one of the thresholds causes the agent to slow down the rate at which the wealth is decumulated, by consuming less every period, which increases the marginal utility of the next increment in wealth.

The ex post value function in the SPE is defined entirely by the utility corresponding to the floor contract. To construct an SPE it suffices to check that the optimal contract designed by $\mathrm{P}$ for zero wealth agents whose savings and effort incentives are defined by this value function, generates exactly the same floor contract. Assumption [B] ensures this is indeed the case.

Proposition 3 Assume [B] holds. Then there exists a simple P-equilibrium with the following properties: there exists $w^{*}>0$ such that

(i) For all $w<w^{*}$, the static $(P)$ optimal contract is offered: $e(w)=e^{*}, y(0)=y^{*}, x(0)=0$ and the resulting floor utility is $V(0)=V^{*} \equiv \frac{e^{*} D^{\prime}\left(e^{*}\right)-D\left(e^{*}\right)}{1-\delta}$.

(ii) For any $w \geq w^{*}$ :

$$
V(w)=B(w)= \begin{cases}\frac{1-\delta^{k}}{1-\delta} u\left(\frac{(1-\delta) w}{1-\delta^{k}}\right)+\delta^{k} V^{*} & \text { if } w \in\left[z_{k-1}, z_{k}\right] \\ \frac{1}{1-\delta} u((1-\delta) w), & \text { if } w>\bar{z}\end{cases}
$$

(iii) there is a poverty trap below $w^{*}$, i.e., $w_{t}<w^{*}$ implies $w_{T}=0$ for all $T>t$ with probability one.

Proof: We first show that $[\mathrm{B}]$ implies that the floor contract must coincide with the static optimal contract.

Lemma 2 If $[B]$ holds, then $e(0)=e^{*}, y(0)=y^{*}$ maximizes $e[R-y]$ subject to $D^{\prime}(e)=$ $\max \{B(y)-B(0), 0\}$ and $y \geq 0$, where $B$ denotes the ex post value function with exit option $V^{*}$. 
Proof: Note that we can restrict the range of feasible values of $y$ to $[0, R]$, since any $y>R$ is strictly dominated by $y=R$. [B] is equivalent to the condition that even with $y=R$, exiting immediately is better than waiting one period, i.e., $u(R)+\delta V^{*}>\frac{1-\delta^{2}}{1-\delta} u\left(\frac{1-\delta}{1-\delta^{2}} R\right)+\delta^{2} V^{*}$. Hence for all $y \in[0, R]$, A will exit at the very next date, and $B(y)=u(y)+B(0)$. So $B(y)-B(0)=u(y)$, and the problem reduces to the static optimal contracting problem. This proves Lemma 2.

The ex post value function $B^{*}$ corresponding to exit option $V=V^{*}$ satisfies $B^{*}(0)=$ $\delta V^{*}<V^{*}=V(0)$. Since $B^{*}$ is continuous and strictly increasing, there exists $w^{*}$ such that $V^{*}=B^{*}\left(w^{*}\right)$.

It remains to check that $V(w)=\max \left\{V^{*}, B^{*}(w)\right\}$ is the ex ante value function for $\mathrm{A}$ generated by the optimal contract $(x(w), y(w), e(w)$ for P, i.e., which for any $w$ maximizes $w-f+e[R-y]-(1-e) x$ subject to $(\mathrm{EIC}): D^{\prime}(e)=\max \left\{B^{*}(y)-B^{*}(x), 0\right\},(\mathrm{APC})$ : $e B^{*}(y)+(1-e) B^{*}(x)-D(e) \geq B^{*}(w),(\mathrm{LL}): y, x \geq 0$, and $(\mathrm{PPC}): w-f+e[R-y]-(1-e) x \geq$ 0 .

We now claim that neither (APC) nor (PPC) binds in this problem if $w<w^{*}$. If $w<w^{*}$ and we drop these two constraints, then the problem reduces to that considered in Lemma 2 above, with $x=0$. It is then evident that the resulting solution satisfies both participation constraints strictly.

On the other hand, at any $w>w^{*}$, note that if there is no feasible non-null contract, then $V(w)=B(w)$. So without loss of generality suppose there is a feasible non-null contract: we have to show that (APC) must bind in any optimal contract. Otherwise, we can drop (APC). Then the problem is the same as when $w<w^{*}$, and we have shown above that in the solution to this problem the agent gets $V^{*}=B\left(w^{*}\right)<B(w)$ and (APC) is violated, a contradiction. This establishes (i) and (ii).

Part (iii) follows from the fact that below $w^{*}$, A must receive the floor contract, which generates end-of-period wealth of 0 or $y^{*}$, both less than $R$, which in turn is less than $z_{1}$, the threshold for immediate exit in the ex post value function $B^{*}$.

The role of assumption $[\mathrm{B}]$ can now be explained. It ensures that $R$, the maximum 
return from the project is less than the first threshold $z_{1}$ required to induce $\mathrm{A}$ to save anything. Since $R$ exceeds the maximum payment that the principal might conceivably make to A (with zero initial wealth) in the event of success, this implies that such an agent will invariably consume his entire end-of-period wealth. This reduces the contracting problem to a static one, so $\mathrm{P}$ will indeed offer such agents the same contract as in a one period setting. In turn this is the floor contract used to construct the ex post value function, so we have constructed an equilibrium.

\subsection{Wealth Dynamics in the Simple P-Equilibrium}

What are wealth dynamics when $w_{0}>w^{*}$ in the SPE?

One obvious case, which includes the case where $u$ is linear:

Proposition 4 Suppose $u$ does not satisfy $M C$ relative to $V^{*}$. Then there is a global poverty trap: $w_{t}=0$ for all $t>1$ with probability one.

Proof: If $u$ does not satisfy MC relative to $V^{*}$, then $c(z)=z$ for all $z$, so the wealth next period is zero, irrespective of current wealth.

With linear utility A has no preference at all for smoothing consumption; in this case the first threshold $z_{1}$ is infinite and A never saves at all, preferring instead to run down his next period wealth to 0 in order to receive the floor contract. Let us consider instead the case where there is enough preference for consumption smoothing that $u$ satisfies MC relative to $V^{*}$, implying that for sufficiently large $z$, the agent will plan to maintain his wealth. Use $\bar{z}^{*}>0$ to denote the maintenance threshold $z_{\text {infty }}$ in the SPE.

Note that if at a wealth level $\tilde{w}>\bar{z}^{*}$, there is no feasible non-null contract, then $x(w)=y(w)=w$, and $\tilde{w}$ is absorbing. The agent is affluent enough, deciding to 'retire' to a stationary consumption, opting out of productive activity. In that case agents sufficiently poor remain locked in poverty for ever, and those sufficiently affluent stay affluent. Initial conditions thus matter at the extremes. What about those starting with intermediate wealths? To analyse this, we make the following assumption. 
Assumption $[\mathbf{C}] \mathcal{W}$, the set of wealth levels for which a non-null feasible contract exists, is an interval, of the form $\left[0, W^{*}\right]$, where $W^{*}$ exceeds $\bar{z}^{*}$, the maintenance threshold in the SPE.

This assumption is satisfied in the following settings. If $u^{\prime}(\infty)$ is sufficiently large, a feasible non-null contract exists at every $w$. Let $u^{\prime}(\infty)$ be denoted by $\beta$. Then since the rate of increase of $B$ is bounded below by $\beta$, it suffices that there exists effort $\hat{e}$ such that $\hat{e} R-f>\frac{\hat{e} D^{\prime}(\hat{e})}{\beta}$, or $\beta>\frac{\hat{e} D^{\prime}(\hat{e})}{\hat{e} R-f}$. Then there is a feasible contract with $\alpha=1, x=w, e=\hat{e}$ for any $w$, and $[\mathrm{C}]$ is satisfied, with $W^{*}=\infty$.

If $u^{\prime}(\infty)=0$ then it is plausible that for $w$ sufficiently large no feasible non-null contract exists. ${ }^{7}$ If a feasible non-null contract exists for all $w \leq \bar{z}^{*}$, and if $\frac{1}{u^{\prime}}$ is convex, then $[\mathrm{C}]$ is satisfied with finite $W^{*}$ (a standard argument used e.g. in Newman (1997) ensures that P's expected profit is decreasing in $w$ ). In this case every $w>W^{*}$ is absorbing.

Given $[\mathrm{C}]$, we now examine properties of the wealth dynamic for an agent starting with initial wealth lying between the poverty trap threshold $w^{*}$ and the 'retirement' threshold $W^{*}$.

Proposition 5 Suppose $[C]$ is satisfied, and $W^{*}$ is finite. Then there exists $\eta>0$ such that:

(i) given any initial wealth $w_{0} \in\left[0, W^{*}\right]$, the agent's wealth $w_{t}$ will converge to 0 in finite time with probability at least $\eta$.

(ii) given any initial wealth $w_{0} \in\left(\bar{z}^{*}, W^{*}\right), w_{t}$ will converge to a level exceeding $W^{*}$ in finite time with probability at least $\eta$.

The proof of this result utilizes the following lemmas, proofs of which are provided in the Appendix.

\footnotetext{
${ }^{7}$ This needs to be verified. The basis for the conjecture is the following. Above the maintenance threshold $B$ is concave in the SPE. Using reasoning analogous to part (i) of lemma 7 below, $x(w)$ will lie above the manitenance threshold for $w$ sufficiently large. Also $\alpha=1$ requires that effort be bounded away from 0 . Then as $w \rightarrow$ infty, providing incentives become infinitely costly.
} 
Lemma 3 For any wealth $w \in\left(w^{*}, W^{*}\right]$, any contract $(x(w), y(w))$ optimal for $P$ at $w$ must satisfy $x(w)<w<y(w)$.

Lemma 4 Consider a sequence of wealth levels $w^{n}$ in $\left[0, W^{*}\right]$ converging to $\hat{w}$, and the corresponding contract sequence in the $\operatorname{SPE}\left(x^{n}, y^{n}, e^{n}\right)$ converging to $(\hat{x}, \hat{y}, \hat{e})$. Then $(\hat{x}, \hat{y}, \hat{e})$ is an optimal contract for $P$ at $\hat{w}$.

Lemma 5 For any $w \in\left[0, W^{*}\right]$, there exists $\epsilon>0$ such that (i) $x(w)<w-\epsilon$, and (ii) $y(w)>w+\epsilon$.

Lemma 6 For any $w \in\left[0, W^{*}\right], e(w)$ is bounded away from 1.

Hence the probability of failure consequent on any $w \in\left[0, W^{*}\right]$ is bounded away from zero. So the probability of any finite number of successive failures is also bounded away from 0, and part (i) of Proposition 5 follows. For part (ii), note that $w>\bar{z}^{*}$ implies that $y(w)>\bar{z}^{*}+\epsilon$, and the starting wealth at the next date following upon a success will be higher than today by at least $\epsilon$. So (ii) follows as long as the success probability is bounded away from zero. But this is ensured by the fact that $y(w)-x(w)$ is bounded below by $2 \epsilon$. This concludes the proof of Proposition 5.

The preceding result establishes that all wealth levels between the poverty and retirement thresholds must be transient. Hence the long run wealth distribution must be polarized between 0 and wealths exceeding $W^{*}$. The same result extends when $W^{*}=\infty$, though the argument is somewhat more involved:

Proposition 6 Suppose $[C]$ is satisfied, and $W^{*}=\infty$. Then from any initial wealth, $w_{t}$ almost surely converges either to 0 or $\infty$. The probability of converging to 0 is positive from any initial wealth. The probability of converging to $\infty$ is positive for any initial wealth above $\bar{z}^{*}$.

We outline the proof here. Define $\underline{e}$ by the condition $\underline{e} R=f$. Then $R>f$ implies that $\underline{e} \in(0,1)$. Next we establish the following Lemma, whose proof is presented in the Appendix. 
Lemma 7 Suppose $W^{*}=\infty$. Then there exists $\bar{W}>\bar{z}^{*}$ and a number $\eta>0$ such that for any $w>\bar{W}$ :

(i) $x(w)>\bar{z}^{*}$

(ii) $e(w) \geq \underline{e}$

(iii) $e(w) y(w)+[1-e(w)] x(w) \geq w+\eta$.

The proof of Proposition 6 then proceeds as follows. Conditional on the event that $w_{t}>\bar{W}$ for all $t$, the strong law of large numbers for Markov processes implies that $w_{t}$ will almost surely converge to $\infty$. Moreover, if initial $w$ is sufficiently high (e.g., higher than some $\tilde{W}>\bar{W})$, then with positive probability $w_{t}$ will stay above $\bar{W}$ for ever for all $t$. For any $w \in[0, \tilde{W}]$, on the other hand, arguments similar to those used in Proposition 5 above will ensure that the probability of converging to 0 is bounded away from zero. Hence for all $w$ the probability of either converging to 0 or $\infty$ is bounded away from zero, so this event must occur almost surely.

We conjecture that the properties established for the simple P-equilibrium are shared by all P-equilibria with continuous value functions.

\section{Where Agents Have All the Bargaining Power}

We now study the 'competitive' case where agents have all the bargaining power. In what follows, we characterize properties satisfied by any A-equilibrium, rather than construct a specific A-equilibrium. Note that arbitrary A-equilibria may involve discontinuous value functions, unlike the particular SPE we analyzed in the previous Section. In this sense the characterization of equilibria in this Section is more general.

We start with a useful lemma, establishing that P's cannot ever earn any positive rents if they have no bargaining power. While the result is intuitive, possible discontinuities in A's value function complicate the argument, since rent extraction by A must be constructed in a way to not result in discontinuous effort changes leading to downward jumps in P's expected profit (that threaten the feasibility of the contract). 
LeMma 8 In any A-equilibrium, PPC binds at every wealth level $w$.

Proof: Suppose not, and there is a contract $\alpha=1, e, y, x$ assigned to wealth $w$ where PPC does not bind.

First consider the case where $B$ is continuous either at $y$ or at $x$. Then we can reduce the transfer to $\mathrm{P}$ at whichever state happens to be a continuity point of $B$. For a sufficiently small transfer, the corresponding effort change induced will be small, so the breakeven constraint will be preserved. The variation will increase A's present value utility, a contradiction.

Next, suppose that $B$ is not continuous at either $y$ or $x$. Then there are three possibilities: (a) $R>y-x$, (b) $R=y-x$, and (c) $R<y-x$. If (a) holds, reduce the transfer to $\mathrm{P}$ in the successful state slightly, so that the inequality $R>y-x$ is maintained. The agent's effort cannot fall in response, so PPC will continue to be respected. Then A's present value utility increases, a contradiction. In case (c) the reverse argument works: the transfer in the unsuccessful state can be reduced slightly to effect an improvement. In case (b), both $y$ and $x$ can be raised in step, so as to preserve the equality of $R$ with $y-x$. Then any change in effort does not affect PPC, while A is rendered better off.

An immediate implication of this result is that all the benefits of incremental initial wealth must accrue to the agent, thus ensuring that the return to saving can never drop to zero as in the case of poor agents in the SPE.

Proposition 7 In any A-equilibrium $V$ is strictly increasing.

Proof: From its definition it is evident that $V$ is nondecreasing. Suppose there exist $w_{1}, w_{2}$ with $w_{2}>w_{1}$ such that $V\left(w_{2}\right)=V\left(w_{1}\right)$. Then $e\left(w_{1}\right), y\left(w_{1}\right), x\left(w_{1}\right)$ is feasible at $w_{2}$, and hence must also be optimal at $w_{2}$. But here PPC does not bind.

The following proposition goes further and provides a lower bound to the rate of return to saving for the agent. This bound is given by the marginal utility of the highest possible level of consumption at the following date. 
Proposition 8 Let $c(y) \equiv y-\delta \omega(y)$ denote the consumption strategy of the agent in any equilibrium. Then at any wealth w:

$$
\lim _{\epsilon \rightarrow 0+} \frac{V(w+\epsilon)-V(w)}{\epsilon} \geq u^{\prime}(\max \{c(y(w)), c(x(w))\}) .
$$

Two corollaries of the preceding result follow. First, in at least one state at the following date consumption must be at least as high as today. Second, in the case where the agent has linear utility, saving his entire end-of-period wealth is a best response.

Proposition 9 (i) If $u$ is strictly concave then in any A-equilibrium the consumptionsaving strategy of the agent must satisfy at any $z$ :

$$
c(z) \leq \max \{c(y(\omega(z))), c(x(\omega(z)))\}
$$

(ii) If $u$ is linear, then it is a best response for the agent to accumulate wealth at the fastest possible rate, i.e., set $\omega(z)=(1+r) z$.

Proof: If (i) is false, $c(z)$ must be positive, so it is feasible for the agent to consume a little bit $(\epsilon>0)$ less. This would cause wealth at the following date to be $\omega(z)+\frac{\epsilon}{\delta}$ instead of $\omega(z)$. It must therefore be the case that for every small $\epsilon>0$ :

$$
\begin{aligned}
u(c(z))-u(c(z)-\epsilon) & \geq \delta\left[V\left(\omega(z)+\frac{\epsilon}{\delta}\right)-V(\omega(z))\right] \\
& \geq \epsilon u^{\prime}(\max \{c(y(\omega(z))), c(x(\omega(z)))\})
\end{aligned}
$$

using Proposition 8. Taking limits with respect to $\epsilon$, we obtain (i). When $u(c)=c$, then Proposition 8 implies that the benefit of increasing next period's wealth by $\frac{\epsilon}{\delta}$ is at least $\epsilon$, the current cost in terms of foregone consumption.

The contrast with the saving strategy in the simple $P$-equilibrium is especially stark in the case when $u$ is linear: in that equilibrium A always wants to run down his wealth to 0 . Here it is always optimal for the agent to consume nothing (though in equilibrium he must 
be consuming somewhere, so must be indifferent between consuming and saving at all such points). Note also that in a static setting the agent needs external finance only if $w<f$. Otherwise if $w>f$, the agent has no need for either credit or insurance from any principal: the first-best can be achieved (with $y(w)=w+R-f, x(w)=w-f$ ). In a dynamic setting, we might initially guess that the same is true for $w$ sufficiently large, with A consuming to ensure wealth maintenance from the end of one period to the beginning of the next. While for lower values of $w$, A is either credit constrained, or faces the possibility of facing such credit constraints in the foreseeable future ( $w$ falling below $f$ ), in which case he saves all his wealth. However, notice that in even if currently $w$ exceeds $f$, it may fall below $f$ at some future date following a string of failures. Anticipation of possible credit constraints in the future may therefore motivate some additional saving even for wealthy self-financing entrepreneurs. Such an equilibrium remains to be constructed (in finite horizon versions of the problem analogues of these do appear). But this intuition suggests a tendency for a perpetual upward drift in the wealth process, especially at low wealth levels. In particular one might expect that the agent will never fall into a poverty trap.

The case of linear utility may be considered too extreme, with the agent have no preferences at all for consumption smoothing. So it is relevant to consider the case where $u$ is strictly concave. Fortunately, we can prove some relevant properties of the wealth dynamics for general concave utility functions, such as the the impossibility of any poverty trap (defined by the property that zero wealth is an absorbing state, i.e., $\omega(y(0))=0=\omega(x(0)))$ :

Proposition 10 If $u$ is strictly concave, there cannot be any A-equilibrium with a poverty trap.

The proof of this relies upon the following extension of Proposition 8, which sharpens the lower bound on the return to saving, to the expected marginal utility of consumption at the following date. This result is of some interest in its own right, as it implies a submartingale property for $\frac{1}{u^{\prime}(c)}$, similar to that in the case of efficient long term insurance contracts.

Proposition 11 (i) Consider any equilibrium and any initial wealth level $w$ for which an optimal contract exists which assigns end-of-period wealth levels $y(w), x(w)$ that are 
both points of (right-) continuity of $B($.$) . Then$

$$
\lim _{\epsilon \rightarrow 0+} \frac{V(w+\epsilon)-V(w)}{\epsilon} \geq\left[e(w) \frac{1}{u^{\prime}(c(y(w)))}+(1-e(w)) \frac{1}{u^{\prime}(c(x(w)))}\right]^{-1}
$$

(ii) Consider any equilibrium and any end-of-period wealth level $z$ which induces end-ofperiod wealth levels at the following date that are both (right-) continuity points of $B$. Then with e denoting $e(\omega(z))$ :

$$
\frac{1}{u^{\prime}(c(z)} \leq e \frac{1}{u^{\prime}(c(y(\omega(z))))}+(1-e) \frac{1}{u^{\prime}(c(x(\omega(z))))}
$$

We turn finally to the intuition concerning upward drift of wealth, as a result of strong saving incentives generated in A-equilibria. This intuition is confirmed irrespective of agents' preferences for consumption smoothing, provided the equilibrium satisfies a mild rightcontinuity property.

Proposition 12 Assume that $u$ is strictly concave, $u^{\prime}(\infty)>0$ and a feasible non-null contract exists at all wealth levels. Then in any A-equilibrium with a right-continuous ex post value function $B$, wealth converges almost surely to $\infty$ from any initial level.

The reasoning behind this result is somewhat involved, but the main idea is the following. Given te concavity of $u$ and $u^{\prime}(\infty)>0$, the martingale convergence theorem implies that consumption converges almost surely. If it converges to a finite limit then the agent must eventually be left with no effort incentives, which would lead to a violation of feasibility as the principals would fail to break even.

What can we say about the case where $u^{\prime}(\infty)=0$ and 'retirement' of sufficiently wealthy agents?

Proposition 13 Assume that a feasible non-null contract does not exist for wealth above some threshold $W^{*}$. Then from any initial wealth $w \in\left[0, W^{*}\right)$, the wealth at some future date will exceed $W^{*}$ with positive probability, in any right-continuous A-equilibrium. 
Hence irrespective of initial conditions every agent must 'retire' with positive probability at some future date. However, it is difficult to ensure that 'retirement' is an absorbing state, so one must entertain the possibility that agents retire for a finite number of periods and then return to productive activity. In this case retirement is an event that will occur infinitely often. Somewhat surprisingly, this last proposition is difficult to extend to its almost sure version: this would require the probability of crossing the threshold at some future date from any initial wealth to be bounded away from zero.

\section{REFERENCES}

Aghion, P. and P. Bolton (1997), "A Theory of Trickle Down Growth and Development," Review of Economic Studies, 64, 151-172.

Atkeson A. and R. Lucas (1992), "On Efficient Distribution with Private Information," Review of Economic Studies, 59, 427-453.

Banerjee, A., P. Gertler and M. Ghatak (1997), "Empowerment and Efficiency: The Economics of Tenancy Reform," mimeo, Department of Economics, M.I.T.

Banerjee, A. and A. Newman (1991), "Risk-Bearing and the Thoery of Income Distribution," Review of Economic Studies, 211-235.

- (1993), "Occupational Choice and the Process of Development," Journal of Political Economy, 101(2), 274-298.

(1994), "Poverty, Incentives and Development," American Economic Review, Papers and Proceedings, 84(2), 211-215.

Bernanke B. and M. Gertler (1990), "Financial Fragility and Economic Performance," Quarterly Journal of Economics, 105, 87-114. 
Bhaduri A. (1973), "Agrarian Backwardness under Semi-Feudalism," Economic Journal, 83, 120-137.

Bowles S. and H. Gintis (1994), "Efficient Redistribution: New Rules for Markets, States and Communities," Working Paper 1994-7, Department of Economics, University of Massachusetts, Amherst.

- (1995), "The Distribution of Wealth and the Assignment of Control Rights," mimeo, Department of Economics, University of Massachusetts, Amherst.

Dutta, B., D. Ray and K. Sengupta (1989), "Contracts with Eviction in Infinitely Repeated Principal Agent Relationships," in Pranab Bardhan (ed.) The Economic Theory of Agrarian Institutions, Clarendon Press, Oxford.

Eswaran M. and A. Kotwal (1986), "Access to Capital and Agrarian Production Organization," Economic Journal.

Galor O. and J. Zeira (1993), "Income Distribution and Macroeconomics," Review of Economic Studies, 60(1), 35-52.

Ghatak M., M. Morelli and T. Sjöstrom (1997), "Dynamic Incentives, Occupational Mobility and the American Dream," mimeo, Department of Economics, Iowa State University.

Green E. (1987), "Lending and the Smoothing of Uninsurable Income," in Contractual Arrangements for Intertemporal Trade, University of Minnesota Press, Minneapolis.

Hoff, K. (1994), "The Second Theorem of the Second Best," Journal of Public Economics, $45,223-242$.

and A. Lyon (1995), "Non-Leaky Buckets: Optimal Redistributive Taxation and Agency Costs," Journal of Public Economics.

Holmstrom, B. and J. Tirole (1994), "Financial Intermediation, Loanable Funds and the Real Sector," Working Paper, Institut D'Economie Industrielle, University of Toulouse. 
Legros, P. and A. Newman (1996), "Wealth Effects, Distribution, and the Theory of Organization," Journal of Economic Theory.

Loury G. (1981), "Intergenerational Transfers and the Distribution of Earnings," Econometrica 49, 843-867.

Mani A. (1998), "Income Distribution and the Demand Constraint," mimeograph, Department of Economics, Vanderbilt University.

Mookherjee, D. (1997a), "Informational Rents and Property Rights in Land," in Property Rights, Incentives and Welfare, J. Roemer (Ed.), Macmillan Press.

- (1997b), "Wealth Effects, Incentives and Productivity," Review of Development Economics.

Newman A. (1997), "Risk-Bearing and Knightian Entrepreneurship," mimeo, Department of Economics, Columbia University.

Phelan C. (1998), "On the Long Run Implications of Repeated Moral Hazard," Journal of Economic Theory, 79, 174-191.

Piketty T. (1997), "The Dynamics of the Wealth Distribution and the Interest Rate with Credit Rationing," Review of Economic Studies.

Ray D. and P. Streufert (1993), "Dynamic Equilibria with Unemployment Due to Undernourishment," Economic Theory.

Rogerson W. (1985), "Repeated Moral Hazard," Econometrica, 53, 69-76.

Shetty Sudhir (1988), "Limited Liability, Wealth Differences and Tenancy Contracts in Agrarian Economies," Journal of Development Economics, 29(1), 1-22.

Thomas J. and T. Worrall (1990), "Income Fluctuations and Asymmetric Information," Journal of Economic Theory, 51, 367-390.

Wang C. (1995), "Dynamic Insurance with Private Information and Balanced Budgets," Review of Economic Studies, 62(4), 577-596. 


\section{APPENDIX}

Proof of Lemma 3: Since $w>w^{*}$, we know $B(w)=V(w)=e(w) B(y(w))+(1-$ $e(w)) B(x(w))-D(e(w))$.

We first claim that $y(w)>x(w)$ and $e(w)>0$. Otherwise $e(w)=0$, and P's expected profit is $w-f-x(w)$. Moreover, $B(w)=B(x(w))$, implying $x(w)=w$. So $\mathrm{P}$ earns a negative expected profit, a contradiction.

Next we claim that $y(w)>w$. Otherwise $x(w)<y(w) \leq w$, implying $V(w)=$ $e(w) B(y(w))+(1-e(w)) B(x(w))-D(e(w))<B(w)$, contradicting (APC).

Finally, suppose $x(w) \geq w$. Then $y(w)>x(w) \geq w$, and A would obtain an expected present value utility of $B(w)$ upon selecting $e=0$. Since $D($.$) is strictly convex and e(w)>0$, A must end up with a present value utility that strictly exceeds $B(w)$, contradicting the property that $V(w)=B(w)$.

Lemma 9 Consider a sequence of wealth levels $w^{n}$ in $\left[0, W^{*}\right]$ converging to $\hat{w}$, and the corresponding contract sequence in the $S P E\left(x^{n}, y^{n}, e^{n}\right)$ converging to $(\hat{x}, \hat{y}, \hat{e})$. Then $(\hat{x}, \hat{y}, \hat{e})$ is an optimal contract for $P$ at $\hat{w}$.

Proof of Lemma 9: Feasibility of the limit contract at $\hat{w}$ follows from continuity of $B$ and $D$. If it is not optimal, there exists $\tilde{x}, \tilde{y}, \tilde{e}$ feasible at $\hat{w}$ which generates for $\mathrm{P}$ a higher expected profit. Define $\tilde{x}^{n}$ by the condition $B\left(\tilde{x}^{n}\right)+\tilde{e} D^{\prime}(\tilde{e})-D(\tilde{e})=V\left(w^{n}\right)$, and $\tilde{y}^{n}$ by the condition $B\left(\tilde{y}^{n}\right)=B\left(\tilde{x}^{n}\right)+D^{\prime}(\tilde{e})$. Then $\left(\tilde{x}^{n}, \tilde{y}^{n}, \tilde{e}\right)$ is feasible at $w^{n}$, and for large enough $n$ will be close enough to $(\tilde{x}, \tilde{y}, \tilde{e})$ to generate a higher profit than $\left(x^{n}, y^{n}, e^{n}\right)$, a contradiction. This establishes Lemma 9.

Lemma 10 For any $w \in\left[0, W^{*}\right]$, there exists $\epsilon>0$ such that (i) $x(w)<w-\epsilon$, and (ii) $y(w)>w+\epsilon$.

Proof of Lemma 10 If (i) is false, we can find a sequence $w^{n}$ in $[0, W]$ such that $w^{n}-$ $x\left(w^{n}\right)$ is converging to 0 . Construct a subsequence along which $\left(x\left(w^{n}\right), y\left(w^{n}\right), e\left(w^{n}\right), w^{n}\right)$ 
converges, say, to $(\hat{x}, \hat{y}, \hat{e}, \hat{w})$. Then by Lemma 9 , the contract $(\hat{x}, \hat{y}, \hat{e})$ is optimal for $\mathrm{P}$ at $\hat{w}$, and $\hat{x}=\hat{w}$, contradicting Lemma 3 . A similar argument establishes (ii).

Lemma 11 For any $w \in\left[0, W^{*}\right]$, e $(w)$ is bounded away from 1.

Proof of Lemma 11 Otherwise, we can find a sequence of wealth levels $w^{n}$ in $\left[0, W^{*}\right]$ for which the corresponding effort levels $e^{n}$ converge to 1 . Since $D^{\prime}\left(e^{n}\right)=B\left(y^{n}\right)-B\left(x^{n}\right), y^{n}$ must converge to $\infty$, and P's profit $w_{n}-f+e^{n}\left[R-y^{n}\right]-\left(1-e^{n}\right) x^{n}$ converges to $-\infty$.

Proof of Lemma 7 If (i) is false, we can construct a sequence $w^{n} \rightarrow \infty$ such that $x^{n}=$ $x\left(w^{n}\right) \leq \bar{z}^{*}$. Then $y^{n} \rightarrow \infty$, otherwise (APC) will be violated for large enough $n$. So $D^{\prime}\left(e^{n}\right)=B\left(y^{n}\right)-B\left(x^{n}\right) \rightarrow \infty$, and $e^{n} \rightarrow 1$. Let $z^{n} \equiv e^{n} y^{n}+\left(1-e^{n}\right) x^{n}$. Then for large $n$, $z^{n}>\bar{z}^{*}$, and

$$
\begin{aligned}
\left(1-e^{n}\right)\left[B\left(z^{n}\right)-B\left(x^{n}\right)\right]+e^{n}\left[B\left(z^{n}\right)-B\left(y^{n}\right)\right] \geq & \left(1-e^{n}\right)\left[B\left(z^{n}\right)-B\left(\bar{z}^{*}\right)\right]+e^{n}\left[B\left(z^{n}\right)-B\left(y^{n}\right)\right] \\
\geq & \left(1-e^{n}\right) B^{\prime}\left(z^{n}\right)\left(z^{n}-\bar{z}^{*}\right)+e^{n} B^{\prime}\left(z^{n}\right)\left(z^{n}-y^{n}\right) \\
& =B^{\prime}\left(z^{n}\right)\left[\left(1-e^{n}\right)\left(z^{n}-\bar{z}^{*}\right)+e^{n}\left(z^{n}-y^{n}\right)\right]
\end{aligned}
$$

the second inequality utilising the fact that $B$ is concave above $\bar{z}^{*}$. Now note that

$$
\lim _{n}\left[\left(1-e^{n}\right)\left(z^{n}-\bar{z}^{*}\right)+e^{n}\left(z^{n}-y^{n}\right)\right]=-\lim _{n}\left(1-e^{n}\right)\left(\bar{z}^{*}-x^{n}\right)=0
$$

since $e^{n} \rightarrow 1$. So

$$
\lim _{n}\left(1-e^{n}\right)\left[B\left(z^{n}\right)-B\left(x^{n}\right)\right]+e^{n}\left[B\left(z^{n}\right)-B\left(y^{n}\right)\right] \geq 0 .
$$

Since $\left.\left(1-e^{n}\right) B\left(x^{n}\right)+e^{n}\right) B\left(y^{n}\right)=B\left(w^{n}\right)+D\left(e^{n}\right)$, it follows that

$$
\lim _{n}\left[B\left(z^{n}\right)-B\left(w^{n}\right)-D\left(e^{n}\right)\right] \geq 0 .
$$

Moreover, $\lim _{n} D\left(e^{n}\right)=\infty$, so

$$
\lim _{n}\left[B\left(z^{n}\right)-B\left(w^{n}\right)\right]=\infty
$$

implying

$$
\lim _{n}\left[z^{n}-w^{n}\right]=\infty
$$


Then P's expected profit which is bounded above by $w_{n}-z^{n}-f+e^{n} R$, must converge to $-\infty$, a contradiction.

To establish (ii), utilize (i) and the strict concavity of $B$ above $\bar{z}^{*}$ to infer that

$$
\begin{gathered}
B(e(w) y(w)+(1-e(w)) x(w))>e(w) B(y(w))+(1-e(w)) B(x(w)) \\
=B(w)+D(e(w)) \\
\geq B(w)
\end{gathered}
$$

so that

$$
e(w) y(w)+(1-e(w)) x(w)>w .
$$

Hence $e(w)<\underline{e}$ implies that P's profit

$$
w-e(w) y(w)-(1-e(w)) x(w)-f+e(w) R<0 .
$$

Finally, (iii) follows from the fact that

$$
B(e(w) y(w)+(1-e(w)) x(w)) \geq B(w)+D(\underline{e})
$$

which completes the proof.

Proof of Proposition 8: We divide the proof into three cases: (a) $R>y-x$; (b) $R<y-x$;

(c) $R=y-x$.

Case (a): Take any positive $\epsilon<e[R-(y-x)]$, where $e$ denotes the effort assigned at $w$. Construct a contract $\left(\tilde{y}_{s}^{n}=y+\frac{\epsilon}{e}, \tilde{y}_{f}^{n}=x\right)$, and let $\tilde{e}^{n}$ denote the associated effort response. Then by construction PPC is satisfied at wealth $w+\epsilon$ by the new contract $\left(\tilde{y}_{s}^{n}, \tilde{y}_{f}^{n}\right)$, if the agent were to continue to select effort $e$. Since $B$ is strictly increasing, the agent's optimal effort response $\tilde{e}^{n} \geq e$. Given $R>\tilde{y}_{s}^{n}-\tilde{y}_{f}^{n}$, PPC must continue to be satisfied at $\tilde{e}^{n}$. Hence the new contract is feasible at wealth $w+\epsilon$. Since the effort $e$ is still available to the agent,

$$
V(w+\epsilon) \geq e B\left(\tilde{y}_{s}^{n}\right)+(1-e) B\left(\tilde{y}_{f}^{n}\right)-D(e)
$$

Note that $B\left(\tilde{y}_{s}^{n}\right)-B(y) \geq u\left(c_{s}+\frac{\epsilon}{e}\right)-u\left(c_{s}\right)$ if $c_{s} \equiv c(y)$ since it is always feasible for the agent to entirely consume any increment in end-of-period wealth. Hence

$$
V(w+\epsilon)-V(w) \geq e\left[u\left(c_{s}+\frac{\epsilon}{e}\right)-u\left(c_{s}\right)\right]
$$




$$
\begin{aligned}
& \geq \epsilon u^{\prime}\left(c_{s}+\frac{\epsilon}{e}\right) \\
& \geq \epsilon u^{\prime}\left(\max \left\{c_{s}, c_{f}\right\}+\frac{\epsilon}{e}\right)
\end{aligned}
$$

where $\left(c_{s}, c_{f}\right)$ denotes $(c(y), c(x))$. The result then follows upon dividing through and taking limits with respect to $\epsilon$.

Case (b): Reverse the argument of the preceding case, and distribute a wealth increase entirely to the unsuccessful rather than successful state to obtain the same conclusion.

Case (c): For arbitrary positive $\epsilon$, construct the new contract $\left(\tilde{y}_{s}^{n}=y+\epsilon, \tilde{y}_{f}^{n}=x+\epsilon\right)$, which will be feasible at wealth $w+\epsilon$ if the agent continues to select $e$. It will be feasible even if he were to change his effort, since $R=y-x$. Using similar reasoning to that used in case (a) above:

$$
\begin{aligned}
V(w+\epsilon)-V(w) & \geq\left[e\left\{u\left(c_{s}+\epsilon\right)-u\left(c_{s}\right)\right\}+(1-e)\left\{u\left(c_{f}+\epsilon\right)-u\left(c_{f}\right)\right.\right. \\
& \geq \epsilon\left[e u^{\prime}\left(c_{s}+\epsilon\right)+(1-e) u^{\prime}\left(c_{f}+\epsilon\right)\right] \\
& \geq \epsilon u^{\prime}\left(\max \left\{c_{s}, c_{f}\right\}+\epsilon\right) .
\end{aligned}
$$

Proof of Proposition 11: (i) By assumption,

$$
V(w)=e B(y)+(1-e) B(x)-D(e)
$$

where $B$ is right-continuous at $y=y(w)$ and $x=x(w)$. Then for small enough $\epsilon>0$, there exist positive incremental payments $\Delta_{y}(\epsilon), \Delta_{x}(\epsilon)$ that solve the following two equations:

$$
\begin{aligned}
e \Delta_{y}+(1-e) \Delta_{x} & =\epsilon \\
B\left(y+\Delta_{y}\right)-B(y) & =B\left(x+\Delta_{x}\right)-B(x)
\end{aligned}
$$

Moreover, $\Delta_{y}(\epsilon)$ and $\Delta_{x}(\epsilon)$ both tend to 0 as $\epsilon \rightarrow 0+$, and so does $\psi(\epsilon) \equiv B\left(y+\Delta_{y}(\epsilon)\right)-$ $B(y)$. By construction, the contract $y+\Delta_{y}(\epsilon), x+\Delta_{x}(\epsilon)$ elicits the same effort response $e$ as the previous contract; hence it is feasible at wealth $w+\epsilon$. Therefore:

$$
V(w+\epsilon)-V(w) \geq \psi(\epsilon)
$$


Since $B(z+\Delta)-B(z) \geq u(c(z)+\Delta)-u(c(z))$, it follows that for $z=y, x$ :

$$
\lim _{\epsilon \rightarrow 0+}\left[\psi(\epsilon)-\Delta_{z}(\epsilon) u^{\prime}(c(z))\right] \geq 0 .
$$

Weighting inequality (18) by the probability of the corresponding outcomes and adding across the two states, it follows that

$$
\lim _{\epsilon \rightarrow 0+}\left[\psi(\epsilon)-\theta^{-1} \epsilon\right] \geq 0
$$

where $\theta$ denotes $\left[e \frac{1}{u^{\prime}(c(y(\omega(z))))}+(1-e) \frac{1}{u^{\prime}(c(x(\omega(z))))}\right]$. (11) then follows from combining $(17)$ and (19), and part (ii) from an argument similar to that in part (i) of Proposition 9.

Proof of Proposition 10: Suppose there is an equilibrium with a poverty trap, which requires that $\omega(y(0))=0=\omega(x(0))$. If $u$ is strictly concave, a standard revealed preference argument implies that $\omega($.$) must be nondecreasing. We also know that y^{*} \equiv y(0)>x^{*} \equiv$ $x(0)$ is necessary for the agent to exert effort and thus satisfy PPC. Hence $\omega(z)=0$ and $B(z)=u(z)+\delta V(0)$ for all $z \in\left[0, y^{*}\right]$. So $B$ is differentiable at $x^{*}$.

If $B$ is right-differentiable at $y^{*}$, then (12) implies that $\frac{1}{u^{\prime}\left(y^{*}\right)} \leq\left[e(0) \frac{1}{u^{\prime}\left(y^{*}\right)}+(1-\right.$ $\left.e(0)) \frac{1}{u^{\prime}\left(x^{*}\right)}\right]$ upon using the hypothesis that $c\left(y^{*}\right)-y^{*}=c\left(x^{*}\right)-x^{*}=0$. Since $\frac{1}{u^{\prime}}$ is strictly increasing, and $e$ less than 1 (otherwise PPC will be violated), this inequality contradicts $y^{*}>x^{*}$.

If $B$ is not right-differentiable at $y^{*}$, then note that the rate of increase of $B$ at $y^{*}$ is bounded below by $u^{\prime}\left(y^{*}\right)$, since it is feasible for the agent to entirely consume all incremental wealth. Now modify the proof of preceding Propositions to infer that the inequality in (9) must be strict at $y^{*}$, which will again generate a contradiction. (If $R \leq y^{*}-x^{*}$ then note that the reasoning employed in the proof of Proposition 8 implies that the rate of increase of $V$ at zero wealth is strictly greater than $u^{\prime}\left(x^{*}\right)$, since at least part of the incremental wealth can be distributed to the agent in the unsuccessful state. And if $R>y^{*}-x^{*}$, the incremental distributions $\Delta_{s}, \Delta_{f}$ constructed in the proof of Proposition 11 on the assumption that $B$ is right-continuous at $y^{*}$ with a slope bounded below by $u^{\prime}\left(y^{*}\right)$, will be feasible even if $B$ is not right-continuous at $y^{*}$. The reason is that the rate of increase of $B$ is then even higher, so in case it is optimal for the agent to change his effort, he would increase it. Since $R>y^{*}-x^{*}$ the increased effort cannot jeopardize PPC.) 
Proof of Proposition 12 Note first that any feasible contract $(y, x, e)$ with $\alpha=1$ at $w$ requires $e>0$ and $y>w$. For if $e=0$, APC requires $B(x) \geq B(w)$, i.e., $x \geq w$. Then $\mathrm{PPC}$ must be violated: $w-f-x \leq-f<0$. And if $y \leq w$ then $e>0$ implies $x<y \leq w$ and APC will be violated.

\section{LEMma 12 In any A-equilibrium:}

(i) $\inf _{w \in \mathcal{W}} e(w)$ is bounded away from 0 if either $\mathcal{W}$, the set of wealth levels for which a feasible nonnull contract exists, is bounded, or if $u^{\prime}(\infty)>0$.

(ii) $\sup _{w \in \mathcal{W}} e(w)$ is bounded away from 1 if $\mathcal{W}$ is bounded.

If (i) is false, we can construct a sequence of wealth levels $w_{n} \rightarrow w$ (where $w$ could possibly be $\infty$ ) and corresponding nonnull contracts $\left(y_{n}, x_{n}, e_{n}\right)$ with $e_{n} \rightarrow 0$. By APC and the incentive constraint, $V\left(w_{n}\right)=B\left(x_{n}\right)+e_{n} D^{\prime}\left(e_{n}\right)-D\left(e_{n}\right) \geq B\left(w_{n}\right)$, so $\lim _{n} V\left(w_{n}\right)=$ $\left.\lim _{n} B\left(x_{n}\right)\right) \geq \lim _{n} B\left(w_{n}\right)$. At the same time, PPC implies $w_{n}-f+e_{n} R-\left(1-e_{n}\right) x_{n} \geq$ $e_{n} y_{n} \geq 0$, or $\lim _{n} w_{n}-\lim _{n} x_{n} \geq f$. Then for arbitrary and small $\epsilon, \eta>0$, for $n$ sufficiently large we will have $B\left(w_{n}\right)-B\left(x_{n}\right)<\eta$ whilst $w_{n}-x_{n}>f-\epsilon$. If $w_{n}$ and $x_{n}$ are bounded above by $\bar{w}$, this contradicts the fact that the rate of increase of $B$ between $x_{n}$ and $w_{n}$ is bounded below by $u^{\prime}(\bar{w})$. Or if $w_{n}$ and $x_{n}$ are unbounded then the rate of increase of $B$ is bounded below by $u^{\prime}(\infty)$.

If (ii) is false we can find a sequence of wealths $w_{n} \rightarrow w$ and corresponding feasible contracts $\left(y_{n}, x_{n}, e_{n}\right)$ in which PPC binds at every $n$, with $e_{n} \rightarrow 1$, so $D^{\prime}\left(e_{n}\right)=B\left(y_{n}\right)-$ $B\left(x_{n}\right) \rightarrow \infty$, which requires $\lim _{n} y_{n}=\infty$. Since PPC binds for each $n$, we have $w_{n}-f+$ $e_{n}\left[R-y_{n}\right]-\left(1-e_{n}\right) x_{n}=0$. This implies that $\lim _{n} w_{n} \geq f-R+\lim _{n} y_{n}=\infty$, contradicting boundedness of $\mathcal{W}$. This proves Lemma 12 .

Now return to the proof of Proposition 12. Since $u^{\prime}(\infty)>0$, Proposition 11 implies that $\frac{1}{u^{\prime}\left(c_{t}\right)}$ forms a submartingale in any right-continuous A-equilibrium, where $c_{t}$ denotes the consumption of the agent at date $t$. Hence $\frac{1}{u^{\prime}\left(c_{t}\right)}$ converges almost surely. Since $u$ is strictly concave, this implies that $c_{t}$ converges almost surely. 
We claim that almost surely $c_{t} \rightarrow \infty$, which will imply that $z_{t} \rightarrow \infty$, and hence that $w_{t} \rightarrow \infty$. (It is readily verfied that $z_{t}$ is bounded if and only if $w_{t}$ is bounded.)

Take any $Z<\infty$ and integer $T>\frac{1}{m}[B(Z)-B(0)]-1$, where $m$ denotes marginal disutility of effort at the lower bound to effort provided by the preceding lemma. Define the event

$$
B(Z) \equiv\left\{c_{t} \quad \text { converges to some } \quad c^{*}<\infty \quad \text { and } \quad z_{t}<Z \text { for all } t\right\}
$$

Note that $z_{t}<Z$ implies existence of some $W$ such that $w_{t}<W$ for all $t$. Using the argument of Lemma 12, we can found bounds $\underline{e}, \bar{e} \in(0,1)$ for effort levels arising in any contract corresponding to wealth in $[0, W]$.

Next, select $\eta \in(0,(1-\delta) m)$. Note that since $u(0)=0$, continuity and concavity of $u$ imply that $u$ is uniformly continuous. Hence we can find $\epsilon>0$ such that $\left|u(c)-u\left(c^{\prime}\right)\right|<\eta$ whenever $\left|c-c^{\prime}\right|<\epsilon$.

Conditional on wealth $w$ at the beginning of date 0 , define for any positive integer $T$ the $T$-step ahead possible realizations of $z, c$ and $w$ under the given $A$-equilibrium in the following manner. Let $n_{t} \in\{s, f\}$ denote the outcome of the project $t$ dates ahead, and let $n^{t}$ denote the history of project outcomes $\left(n_{t}, n_{t-1}, \ldots, n_{0}\right)$ between dates 0 and $t$. The contract itself specifies $z^{0}\left(n_{0}, w\right)=y(w)$ if $n_{0}=s$ and $x(w)$ otherwise. Using the equilibrium consumption strategy this enables us to work out $c^{0}\left(n_{0}, w\right)=c\left(z^{0}\left(n_{0}, w\right)\right)$, and the wealth at the beginning of next date: $w^{1}\left(n_{0}, w\right)=\delta^{-1}\left[z^{0}\left(n_{0}, w\right)-c^{0}\left(n_{0}, w\right)\right.$. Proceeding in this fashion we can find the $T$-step ahead realizations as functions of the history of the outcomes of the project between 0 and $T: z^{T}\left(n^{T}, w\right), c^{T}\left(n^{T}, w\right), w^{T}\left(n^{T-1}, w\right)$.

Define $\mathcal{C}(w, T)=\left\{c \mid c=c^{t}\left(n^{t}, w\right), t \leq T\right\}$, the set of possible realizations of consumptions $T$-steps ahead.

Then define the event

$$
\mathcal{A}(Z) \equiv\left\{e_{t} \in(\underline{e}, \bar{e}) \quad \text { for all } \quad t \quad \text { and } \quad \lim _{t \rightarrow \infty} \operatorname{diam\mathcal {C}}\left(w_{t}, T\right)=0\right\}
$$

Lemma 13 For any $Z$ :

$$
\operatorname{Prob}[\mathcal{A}(Z) \mid B(Z)]=1
$$


Proof of Lemma 13. Define the event $\mathcal{A}_{1}(Z) \equiv\left\{e_{t} \in(\underline{e}, \bar{e})\right.$ for all $\left.t\right\}$.Note that $\operatorname{Prob}\left[\mathcal{A}_{1}(Z) \mid B(Z)\right]=1$, implying that $\operatorname{Prob}[\mathcal{A}(Z) \mid B(Z)]=\operatorname{Prob}\left[\mathcal{A}(Z) \mid \mathcal{A}_{1}(Z) \cap B(Z)\right]$. We claim that this latter probability equals one. If this is false there is a positive measure of paths for each of which the following is true. There is $\zeta>0$ and an infinite sequence of dates such that for any date $t$ in this sequence, we can find a possible consumption $c^{k}\left(n^{k}, w_{t}\right)$, $k \leq T$ steps ahead such that $\left|c^{k}\left(n^{k}, w_{t}\right)-c^{*}\right|>\zeta$. Since $e_{t}$ is bounded away from zero and one, every $k$-step outcome history has probability bounded away from zero, and almost surely will occur infinitely often. Then almost surely $c_{k+t}$ will equal $c^{k}\left(n^{k}, w_{t}\right)$ for an infinite sequence of dates $k+t$. So there will be a positive measure of paths along which $c_{t}$ fails to converge, contradicting the martingale convergence theorem.

Lemma 14 For any $Z$ :

$$
\operatorname{Prob}[\mathcal{A}(Z) \cap B(Z)]=0
$$

Proof of Lemma 14. The proof rests on the following claim.

Claim. Let $s^{t}$ (resp. $f^{t}$ ) denote $t$-step histories in which the project results in a success (resp. failure) in every period. Then for any $Z$ :

$\operatorname{Prob}\left[B\left(z^{T}\left(s^{T}, w_{t}\right)\right)-B\left(z^{T}\left(f^{T}, w_{t}\right)\right)>m(T+1) \quad\right.$ for all $\quad t>t^{*} \quad$ for some $\left.\quad t^{*} \mid \mathcal{A}(Z) \cap \mathcal{B}(Z)\right]=1$

Consider any path in $\mathcal{A}(Z) \cap \mathcal{B}(Z)$, and select $t^{*}$ such that $\operatorname{diam\mathcal {C}}\left(w_{t}, T\right)<\frac{\epsilon}{2}$ for all $t>t^{*}$. Note that along any such path, $e_{t}>\underline{e}$ at all $t$; hence the effort incentive constraint implies $B\left(z^{1}\left(s^{1}, w_{t}\right)\right)-B\left(z^{1}\left(f^{1}, w_{t}\right)\right)>m$ for all $t$. So the inequality

$$
B\left(z^{\tilde{T}}\left(s^{\tilde{T}}, w_{t}\right)\right)-B\left(z^{\tilde{T}}\left(f^{\tilde{T}}, w_{t}\right)\right)>m(\tilde{T}+1)
$$

holds for $\tilde{T}=1$ for all $t$. We shall show that if it holds for $\tilde{T}-1$ it holds for $\tilde{T}$ as well.

Use $z^{S}$ and $z^{S S}$ to denote $z^{\tilde{T}-1}\left(s^{\tilde{T}-1}, w_{t}\right), z^{\tilde{T}}\left(s^{\tilde{T}}, w_{t}\right)$ respectively. Similarly use $z^{F}$ and $z^{F F}$ to denote $z^{\tilde{T}-1}\left(f^{\tilde{T}-1}, w_{t}\right), z^{\tilde{T}}\left(f^{\tilde{T}}, w_{t}\right)$ respectively. And use $z^{F S}, z^{S F}$ to denote $z^{\tilde{T}}\left(f, s^{\tilde{T}-1}, w_{t}\right)$ and $z^{\tilde{T}}\left(s, f^{\tilde{T}-1}, w_{t}\right)$ respectively. 
Next note that

$$
V\left(\omega\left(z^{S}\right)\right)-V\left(\omega\left(z^{F}\right)\right)=\frac{1}{\delta}\left[B\left(z^{S}\right)-B\left(z^{F}\right)\right]-\frac{1}{\delta}\left[u\left(c\left(z^{S}\right)\right)-u\left(c\left(z^{F}\right)\right)\right]
$$

By the induction hypothesis $B\left(z^{S}\right)-B\left(z^{F}\right)>\tilde{T} m$. Moreover, since $t>t^{*}$, we have ensured by construction that $\frac{1}{\delta}\left[u\left(c\left(z^{S}\right)\right)-u\left(c\left(z^{F}\right)\right)\right]<\frac{1}{\delta} \eta$. Since $\eta<(1-\delta) m$ :

$$
V\left(\omega\left(z^{S}\right)\right)-V\left(\omega\left(z^{F}\right)\right) \geq \frac{1}{\delta}[\tilde{T} m-\eta]>\tilde{T} m
$$

Since

$$
\begin{aligned}
V\left(\omega\left(z^{S}\right)\right) & =\max _{e}\left[e B\left(z^{S S}\right)+(1-e) B\left(z^{F S}\right)-D(e)\right] \\
V\left(\omega\left(z^{F}\right)\right) & =\max _{e}\left[e B\left(z^{S F}\right)+(1-e) B\left(z^{F F}\right)-D(e)\right]
\end{aligned}
$$

it is evident that $V\left(\omega\left(z^{S}\right)\right)-V\left(\omega\left(z^{F}\right)\right)>\tilde{T} m$ implies that $\max \left\{B\left(z^{S S}\right)-B\left(z^{F S}\right), B\left(z^{S F}\right)-\right.$ $\left.B\left(z^{F F}\right)\right\}>\tilde{T} m$. Since $B\left(z^{S S}\right)-B\left(z^{F S}\right) \geq m$ and $B\left(z^{S F}\right)-B\left(z^{F F}\right) \geq m$, it then follows that $B\left(z^{S S}\right)-B\left(z^{F F}\right)>(\tilde{T}+1) m$, establishing the Claim.

We are now in a position to prove Lemma 14. Consider any path along which consumption converges to some $c^{*}<\infty$, and $z_{t}$ is bounded above by $Z$. Then $B\left(z_{t}\right)$ is bounded above by $B(Z)$. Recall that we selected $T>\frac{1}{m}[B(Z)-B(0)]-1$. The Claim above implies then that

$$
B\left(z^{T}\left(s^{T}, w_{t}\right)\right)>m(T+1)+B\left(z^{T}\left(f^{T}, w_{t}\right)\right)>\geq m(T+1)+B(0)>B(Z)
$$

i.e., that $z^{T}\left(s^{T}, w_{t}\right)$ exceeds the upper bound $Z$ for all $t>t^{*}$. Since given event $\mathcal{A}(Z)$, a string of $T$ successive successes will almost surely occur infinitely often, the result of Lemma 14 follows.

Combining the results of Lemmas 13 and 14 it follows that $\operatorname{Prob}[\mathcal{B}(Z)]=0$ for any $Z$. If we define the event $\mathcal{B} \equiv \cup_{k=1}^{\infty} \mathcal{B}(k)=\lim _{k} \mathcal{B}(k)$ that $z_{t}$ is bounded while $c_{t}$ converges to a finite limit, this implies that $\operatorname{Prob}[\mathcal{B}]=0$. Hence if consumption converges to a finite limit, almost surely $z_{t} \rightarrow \infty$; hence $w_{t} \rightarrow \infty$. Proposition 12 then follows from the observation 
that it is impossible for consumption to converge to finite limit $c^{*}$ while wealth converges to infinity. Using Proposition 8 this would require the asymptotic rate of increase of $V$ to be bounded below by $u^{\prime}\left(c^{*}\right)$. But $V$ is bounded above by the value function $\tilde{V}$ corresponding to the case where effort disutility function is identically zero, whose asymptotic rate of increase equals $u^{\prime}(\infty)<u^{\prime}\left(c^{*}\right)$, and we obtain a contradiction.

Proof of Proposition 13 is analogous to that of the previous Proposition. If the result is false, then wealth and consumption is bounded with probability one, which ensures that $\frac{1}{u^{\prime}\left(c_{t}\right)}$ again forms a submartingale, so $c_{t}$ converges almost surely. Lemma 12 ensures that effort is bounded away from zero and one, so all finite step histories will occur infinitely often with probability one. 